\title{
Proximal vortex cycles and vortex nerve structures. Non-concentric, nesting, possibly overlapping homology cell complexes
}

\author{
James F. Peters ${ }^{a b^{*}}$ \\ ${ }^{a}$ Computational Intelligence Laboratory, University of Manitoba, WPG, MB, R3T 5V6, Canada \\ ${ }^{\mathrm{b}}$ Department of Mathematics, Faculty of Arts and Sciences, Adiyaman University, 02040 Adiyaman, Turkey \\ *Corresponding author E-mail: james.peters3@umanitoba.ca
}

\author{
Article Info \\ Keywords: Connectedness Proximity, \\ CW Topology, Vortex Cycle, Vortex \\ Nerve \\ 2010 AMS: 68U05, 55R40, 54E05. \\ Received: 18 May 2018 \\ Accepted: 17 July 2018 \\ Available online: 30 September 2018
}

\begin{abstract}
This article introduces proximal planar vortex 1-cycles, resembling the structure of vortex atoms introduced by William Thomson (Lord Kelvin) in 1867 and recent work on the proximity of sets that overlap either spatially or descriptively. Vortex cycles resemble Thomson's model of a vortex atom, inspired by P.G. Tait's smoke rings. A vortex cycle is a collection of non-concentric, nesting 1-cycles with nonempty interiors (i.e., a collection of 1-cycles that share a nonempty set of interior points and which may or may not overlap). Overlapping 1-cycles in a vortex yield an Edelsbrunner-Harer nerve within the vortex. Overlapping vortex cycles constitute a vortex nerve complex. Several main results are given in this paper, namely, a Whitehead CW topology and a Leader uniform topology are outcomes of having a collection of vortex cycles (or nerves) equipped with a connectedness proximity and the case where each cluster of closed, convex vortex cycles and the union of the vortex cycles in the cluster have the same homotopy type.
\end{abstract}

\section{Introduction}

This paper introduces vortex cycles restricted to the Euclidean plane. Each vortex cycle $A$ (denoted by vcyc $A$ ) is a collection of nonconcentric, nesting 1-cycles with nonempty interiors. A 1-cycle is a finite, collection of vertices (0-cells) connected by oriented edges (1-cells) that define a simple, closed path so that there is a path between any pair of vertices in each 1-cycle. A path is simple, provided it has no self-intersections.

Let $\mathrm{vcyc} A$ be a finite region of the Euclidean plane (denoted by $\mathbb{R}^{2}$ ). Also, let bdy $(\mathrm{vcyc} A)$ be a set of boundary points of vcyc $A$. Then, for every vortex cycle, there is a collection of functions $f: \operatorname{bdy}(\operatorname{vcyc} A) \longrightarrow \mathbb{R}^{2}$ such that each function maps a vcyc $A$ boundary point to an interior fixed point shared by the 1-cycles in the vortex. The physical analogue of a vortex cycle is a collection of non-concentric, nesting equipotential curves in an electric field [3, §5.1, pp. 96-97]. This view of vortex cycles befits a proximal physical geometry approach to the study of vortices in the physical world [37].

Oriented 1-cycles by themselves in vortex cycles are closed braids [5] with nonempty interiors. The study of vortex cycles and their spatial as well as descriptive proximities is important in isolating distinctive shape properties such as vertex area, cycle overlap count, hole count, nerve count, perimeter, diameter over surface shape sub-regions. A finite, bounded planar shape A (denoted by $\operatorname{sh} A$ ) is a finite region of the Euclidean plane bounded by a simple closed curve and with a nonempty interior [40]. In effect, a vortex cycle is a system of shapes within a shape $^{1}$

\footnotetext{
${ }^{1}$ Many thanks to M.Z. Ahmad for pointing this out.
} 
The geometry of vortex cycles is related to the study shape signatures [39], the study of Edelsbrunner-Harer nerves on tessellated, finite, bounded planar regions [32] and the geometry of photon vortices by N.M. Litchinitser [26], overlapping vortices by E. Adelberger, G. Dvali and A. Gruzinov [14], vortex properties of photons and electromagnetic vortices formed by photons by I.V. Dzedolik [13] and vortex atoms introduced by Kelvin [24].

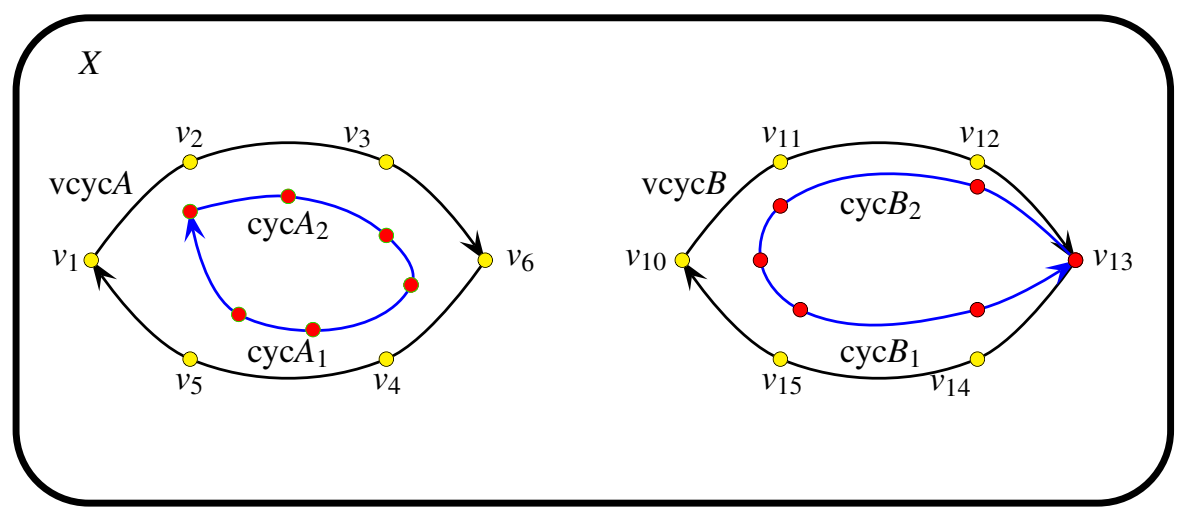

Figure 1: Pair of Two Different Vortex Cycles

Overlapping 1-cycles in a vortex constitute an Edelsbrunner-Harer nerve within the vortex. Let $F$ be a finite collection of sets. An Edelsbrunner-Harer nerve [15, §III.2, p. 59] consists of all nonempty subcollections of $F$ (denoted by Nrv $F$ ) whose sets have nonempty intersection, i.e.,

$$
\operatorname{Nrv} F=\{X \subseteq F: \bigcap X \neq \emptyset\} \text { (Edelsbrunner-Harer Nerve). }
$$

Example 1. Two Forms of Vortex Cycles.

Two different vortex cycles $v c y c A, v c y c B$ are shown in Fig. 1. Vortex vcycA contains a pair of non-overlapping 1-cycles cycA $A_{1}$, cycA $A_{2}$. By contrast, vortex $v c y c B$ in Fig. 1 contains a pair of overlapping 1-cycles $c y c B_{1}, c y c B_{2}$ with a common vertex, namely, $v_{13}$. Let $F$ be a collection of sets of edges in $c y c B_{1}, c y c B_{2}$. The pair of 1-cycles in vortex vcycB constitute an Edelsbrunner-Harer nerve, since $c y c B_{1} \cap c y c B_{2}=v_{13}$, i.e., the intersection of 1-cycles $c y c B_{1}, c y c B_{2}$ is nonempty. The edges of the cycles in both forms of vortices define closed convex curves.

A number of simple results for vortex cycles come from the Jordan Curve Theorem.

Theorem 1. [Jordan Curve Theorem [23]].

A simple closed curve lying on the plane divides the plane into two regions and forms their common boundary.

Proof. For the first complete proof, see O. Veblen [50]. For a simplified proof via the Brouwer Fixed Point Theorem, see R. Maehara [28]. For an elaborate proof, see J.R. Mundres [29, §63, 390-391, Theorem 63.4].

Lemma 1. A finite planar shape contour separates the plane into two distinct regions.

Proof. The boundary of each planar shape is a finite, simple closed curve. Hence, from Theorem 1, a finite, planar shape separates the plane into two regions, namely, the region outside the shape boundary and the region in the shape interior.

Theorem 2. A finite planar vortex cycle is a collection of non-concentric, nesting shapes within a shape.

Proof. Each 1-cycle in a finite planar vortex cycle is a simple, closed curve. By definition, a vortex cycle is a collection of non-concentric 1-cycles nesting within a 1-cycle, each with a nonempty interior. From Theorem 1, each vortex 1-cycle separates the plane into two regions. Hence, from Lemma 1, a finite planar vortex is a collection of planar shapes within a shape.

A darkened region in a planar shape represents a hole in the interior of the shape. In cellular homology, a cell complex $K$ is a Hausdorff space and a sequence of subspaces called skeletons [8] (also called a CW complex or Closure-finite Weak topology complex [22]). Minimal planar skeletons are shown in Table 1.

Table 1 includes a $K_{1.5}$ skeleton, which is a filled triangle with a 2-hole in its interior. The fractional dimension of a $K_{1.5}$ skeleton signals the fact such a skeleton has a partially filled interior, punctured with one or more holes. A 2-hole is a planar region with a boundary and an empty interior. For example, a finite simple, closed curve that is the boundary of a planar shape defines a 2-hole.

For a recent graphics study of polygons with holes in their interiors, see H. Boomari, M. Ostavari and A. Zarei [20]. Also, from Table 1, it is apparent from the grey shading that a $K_{2}$ skeleton is the intersection of three half planes that form a filled triangle. Similarly, a 6-sided 1 -cycle such as cyc $A_{2}$ in vortex cycle vcyc $A$ in Fig. 1 is the intersection of six half planes that construct a 6-gon with a nonempty interior. Recall that a polytope is the intersection of finitely-many closed half planes [53]. In general, a 1-cycle is an $n$-sided polytope that is the intersection of $n$ half planes. 


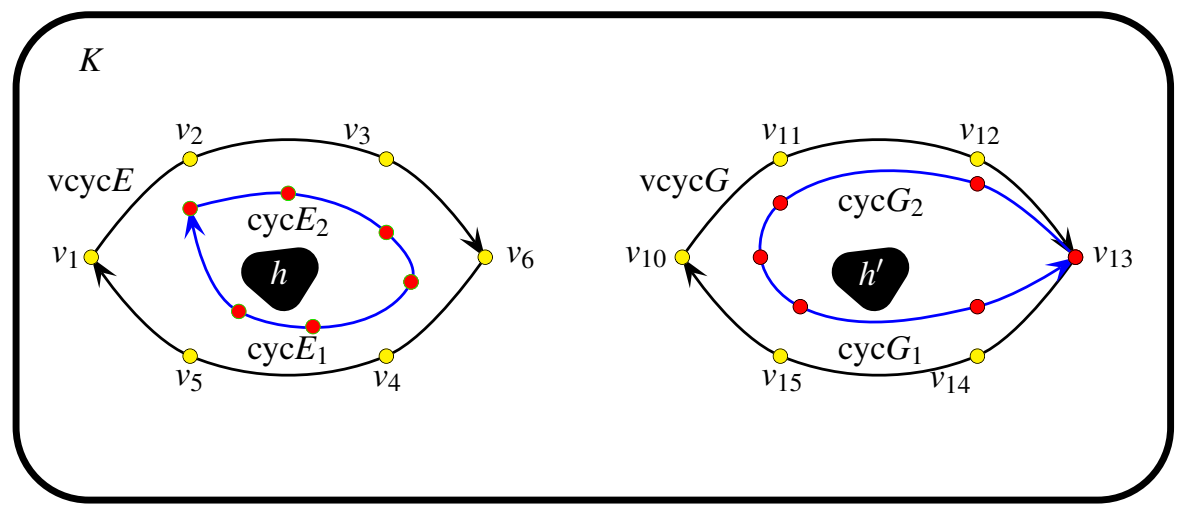

Figure 2: Pair of Two Different Vortex Cycles With Holes

Table 1: Minimal Planar Cell Complex Skeletons

\begin{tabular}{|c|c|c|c|}
\hline Minimal Skeleton & $K_{i}, i=0,1,1.5,2$ & Planar Geometry & Interior \\
\hline - & $K_{0}$ & Vertex & nonempty \\
\hline & $K_{1}$ & Line segment & nonempty \\
\hline & $K_{1.5}$ & Partially filled triangle containing a 2-hole & nonempty \\
\hline & $K_{2}$ & Filled triangle & nonempty \\
\hline
\end{tabular}

Problem 1. How many 2-holes are needed to destroy a 1-cycle, making it a shape boundary with an empty interior?

Problem 2. The diameter of a 2-hole is the maximum distance between a pair of points on the boundary of the 2-hole. What is the diameter of a 2-hole in a filled, planar n-sided polytope that destroys a 1-cycle, making it a shape boundary with an empty interior?

\section{Example 2. Vortex Cycles with Holes.}

Two different vortex cycles with holes are shown in Fig. 2, namely, vcycE, vcycG. The vortex cycle vcycE is an example of a 1-cycle within a 1-cycle (i.e., $c y c E_{2}$ within $\left.c y c E_{1}\right)$ in which $c y c E_{2}$ has a 2-hole $h$ in its interior. The vortex cycle vcycG is an example of intersecting 1-cycles (i.e., cyc $G_{2}$ within cyc $G_{1}$ ) that form a vortex nerve in which cyc $G_{2}$ has a 2-hole $h^{\prime}$ in its interior. In both cases, each inner 1-cycle is in the interior of an outer 1-cycle. Hence, the 2-hole in the interior of the inner 1-cycle is common to the interiors of both 1-cycles in each vortex. For example, 2-hole $h^{\prime}$ in vortex nerve vcycG is common to both of its 1-cycles.

Theorem 3. Let $K$ be a collection of skeletons in a planar cell complex.

$1^{\circ}$ In $K$, skeletons $K_{0}, K_{1}, K_{2}$ are planar shapes.

$2^{o}$ A $K_{1.5}$ skeleton is a planar shape.

$3^{\circ}$ A 1-cycle cycA with a hole $h \in \operatorname{int}(c y c A)$ that is a proper subset in the interior of cycA is a planar shape.

$4^{\circ}$ A planar vortex cycle with a hole is a collection of overlapping 1-cycles, each with a hole.

$5^{\circ}$ A planar vortex cycle with a hole is a collection of concentric planar shapes.

Proof.

$1^{o}$ : By definition, every member of $K$ is a skeleton. Each of the skeletons $K_{0}, K_{1}, K_{2}$ has a boundary with nonempty interior. Hence, these skeletons are planar shapes.

$2^{o}$ : By definition, a $K_{1.5}$ skeleton is a closed 3-sided polytope that has a nonempty interior with a hole. That is, let $h \in \operatorname{int}(c y c A)$ be a 2-hole that is a proper subset in the interior of a $K_{1.5}$ skeleton. In that case, the nonempty part of interior of the $K_{1.5}$ skeleton int $(c y c A)$ equals $\operatorname{int}(c y c A) \backslash h$. In effect, $c y c A$ is a planar shape.

$3^{o}$ : That a 1-cycle $c y c A$ with a hole that is a proper subset in the interior of $c y c A$ is a planar shape, follows from Part 2.

$4^{o}$ : Immediate from Part 3.

$5^{\circ}$ : Immediate from Part 3 and Theorem 2.

Let $\left(K, \delta_{\Phi}\right)$ be a collection of planar vortex cycles equipped with a descriptive proximity $\delta_{\Phi}[6, \S 4]$, [35, $\left.\S 1.8\right]$, based on the descriptive intersection $\underset{\Phi}{\bigcap}$ of nonempty sets $A$ and $B[33, \S 3]$. With respect to vortex cycles vcyc $E$, vcyc $G$ in $K$, for example, we consider vcyc $E \cap{ }_{\Phi} \mathrm{vcyc} G$, i.e., the set of descriptions common to a pair of vortex cycles. A vortex cycle description is a mapping $\Phi: 2^{K} \longmapsto \mathbb{R}^{n}$ (an $n$-dimensional 
feature space). For each given vortex cycle vcyc $E$, find all vortex cycles vcyc $G$ in $K$ that have nonempty descriptive intersection with $\operatorname{vcyc} E$, i.e., $\operatorname{cyc} A \underset{\Phi}{\bigcap} \operatorname{cyc} B \neq \emptyset$ such that $\Phi(\operatorname{vcyc} G)=\Phi(\operatorname{vcyc} E)$. This results in a Leader uniform topology on $H_{1}$ [25].

\title{
2. Preliminaries
}

This section briefly presents the axioms for connectedness, strong and descriptive proximity. A nonempty set $P$ is a proximity space, provided the closeness or remoteness of any two subsets in $P$ can be determined.

\subsection{Cech Proximity Space}

A proximity space $P$ is sometimes called a $\delta$-space [44], provided $P$ is equipped with a relation $\delta$ that satisfies, for example, the following Cech axioms for sets $A, B, C \in 2^{P}[48, \S 2.5$, p. 439].

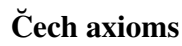 \\ P1 All subsets in $P$ are far from the empty set. \\ P2 $A \delta B \Longrightarrow B \delta A$, i.e., $A$ close to $B$ implies $B$ is close to $A$. \\ $\mathrm{P} 3 A \delta(B \cup C) \Leftrightarrow A \delta B$ or $A \delta C$. \\ P4 $A \cap B \neq \emptyset \Longrightarrow A \delta B$.
}

A space $P$ equipped with the $\breve{C}$ ech proximity (denoted by $(P, \delta)$ ) is called a C̆ech proximity space. We adopt the convention for a proximity metric $\delta: 2^{P} \times 2^{P} \longrightarrow\{0,1\}$ introduced by Ju. M. Smirnov [44, $\S 1$, p. 8]. We write $\delta(A, B)=0$, provided subsets $A, B \in 2^{P}$ are close and $\delta(E, H)=1$, provided subsets $A, B \in 2^{P}$ are not close, i.e., there is a non-zero distance between $E$ and $H$. Let $A, B, C \in 2^{P}$. Then a proximity space satisfies the following properties.

\section{Smirnov Proximity Space Properties}

Q1 If $A \subseteq B$, then for any $C, \delta(A, C) \geq \delta(B, C)$.

Q2 Any sets which intersect are close.

Q3 No set is close to the empty set.

In a C̆ech proximity space, Smirnov proximity space property Q3 is satisfied by axiom $P 1$ and property $Q 2$ is satisfied by axioms P2-P4, i.e., any subsets of $\mathrm{P}$ are close, provided the subsets have nonempty intersection. That is, $A$ close to $B$ implies $B$ is close to $A$ (axiom $\mathrm{P} 2$ ). Similarly, $A$ close to $B \cup C$ implies $A$ is close to $B$ or $A$ is close to $C$ (axiom P3) or $A$ is close to $B \cap C$ (axiom P4). Let $A \cap C=\emptyset$. Then $\delta(A, C)=1$, since $A$ has no points in common with $C$. Similarly, assume $B \cap C=\emptyset$. Then, $\delta(B, C)=1$, since $B$ and $C$ have no points in common. Hence, property Q1 is satisfied, since

$$
\delta(A, C)=\delta(B, C)=1 \Rightarrow \delta(A, C) \geq \delta(B, C)
$$

For $A \subset B$ and $C \subset B$, we have $\delta(A, C)=0$, since $A$ and $C$ have points in common. Similarly, $\delta(B, C)=0$. Hence, $\delta(A, C)=\delta(B, C)=$ $0 \Rightarrow \delta(A, C) \geq \delta(B, C)$.

\subsection{Connectedness Proximity Space}

Let $K$ be a collection of skeletons in a planar cell complex and let $A, B, C$ be subsets containing skeletons in $K$ equipped with the relation $\stackrel{c o n n}{\delta}$. The pair $A, B$ is connected, provided $A \cap B \neq \emptyset$, i.e., there is a skeleton in $A$ that has at least one vertex in common a skeleton in $B$. Otherwise, $A$ and $B$ are disconnected.

Let $X$ be a nonempty set and let $A, B \in 2^{X}$, nonempty subsets in the collection of subsets $2^{X} . A$ and $B$ are mutually separated, provided $A \cap B=\emptyset$, i.e., $A$ and $B$ have no points in common [52, $\$ 26.4$, p. 192]. From the notion of separated sets, we obtain the following result for connected spaces.

Theorem 4. [52]

If $X=\bigcup_{n-1}^{\infty} X_{n}$, where each $X_{n} \in 2^{X}$ is connected and $X_{n-1} \cap X_{n} \neq \emptyset$ for each $n \geq 2$, then space $X$ is connected.

Proof. The proof is given by S. Willard [52, §26.4, p. 193]. For a new kind of connectedness in which nonempty intersection is replaced by strong nearness, see C. Guadagni [19, p. 72] and in J.F. Peters [35, §1.16].

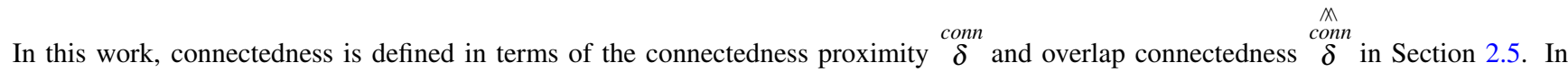
both cases, nonempty intersection is replaced by a connectedness proximity in the study of connected cell complex spaces populated by connected skeletons. For connected sets $A, B \subset K$, we write $A \stackrel{c o n n}{\delta} B$. In effect, for each pair of skeletons $A, B$ in $K, A \stackrel{\text { conn }}{\delta} B$, provided there is a path between at least one vertex in $A$ and one or more vertices in $B$. A path is sequence of edges between a pair of vertices. 
Equivalently, $A \cap B \neq \emptyset$ implies $A \stackrel{\text { conn }}{\delta} B$. If the sets of skeletons $A, B \in K$ are separated (i.e., $A, B$ have no vertices in common), we write $A \stackrel{\text { conn }}{\delta} B$. This view of connectedness

Then the C̆ech axiom P4 is replaced by

P4conn $A \cap B \neq \emptyset \Leftrightarrow A \stackrel{\operatorname{conn}}{\delta} B$

By replacing $\delta$ with $\stackrel{\text { conn }}{\delta}$ in the remaining C̆ech axioms, we obtain

\section{Connectedness proximity axioms.}

P1conn $A \cap B=\emptyset \Leftrightarrow A \stackrel{\text { conn }}{\delta} B$, i.e., the sets of skeletons $A$ and $B$ are not close ( $A$ and $B$ are far from each other).

P2conn $A \stackrel{\text { conn }}{\delta} B \Longrightarrow B \stackrel{\text { conn }}{\delta}$ A, i.e., $A$ close to $B$ implies $B$ is close to $A$.

$\mathrm{P} 3 \operatorname{conn} A \stackrel{\text { conn }}{\delta}(B \cup C) \Longrightarrow A \stackrel{\text { conn }}{\delta} B$ or $A \stackrel{\text { conn }}{\delta} C$.

P4conn $A \cap B \neq \emptyset \Leftrightarrow A \stackrel{\text { conn }}{\delta} B$ (Connectedness Axiom).

A connectedness proximity space is denoted by $(K, \stackrel{c o n n}{\delta})$. For $A, B \in K$, the Smirnov metric $\delta(A, B)=0$ means that there is a path between any two vertices in $A \cup B$ and $\delta(A, B)=1$ means that there is no path between any two vertices in $A \cup B$.

Lemma 2. Let $K$ be a collection of skeletons in a planar cell complex equipped with the relation $\stackrel{\operatorname{conn}}{\delta}$. Then $A \stackrel{\operatorname{conn}}{\delta} B$ implies $A \cap B \neq \emptyset$.

Proof. $A \stackrel{\text { conn }}{\delta} B$, provided there is a path between any pair of vertices in skeletons $A$ and $B$, i.e., $A, B$ are connected, provided there is a vertex common to $A$ and $B$. Consequently, $A \cap B \neq \emptyset$.

Lemma 3. Let $K$ be a connectedness space containing a collection of skeletons in a planar cell complex equipped with the relation ${ }^{c o n n}$. The space $K$ is a proximity space.

Proof. Let $A, B, C \in K$. Smirnov proximity space property Q3 is satisfied by axiom $P 1$ conn and property $Q 2$ is satisfied by axioms $\mathbf{P} 2$ connP4conn, i.e., any sets of skeletons that are close, are connected. Let $C \subset A \cup B(C$ is part of the skeleton $A \cup B \in K)$. For any vertex $p$ in $A$ or $B$, there is a path between $p$ and any vertex $q \in C$. Then $A{ }^{c o n n} \delta$ and $B \stackrel{c o n n}{\delta} C$. Consequently, $\delta(A, C)=0=\delta(B, C)$, Hence, $\delta(A, C) \geq \delta(B, C)$. If $(A \cup B) \cap C=\emptyset$ (the skeletons in $A$ and $B$ have no vertices in common with $C$ ), then $\delta(A, C)=1=\delta(B, C)$ and $\delta(A, C) \geq \delta(B, C)$. From axiom P4conn, we have

$$
(A \cup B) \stackrel{\operatorname{conn}}{\delta} C \Leftrightarrow(A \cup B) \cap C=\emptyset \Leftrightarrow \delta(A, C)=1=\delta(B, C) \Rightarrow \delta(A, C) \geq \delta(B, C) .
$$

Smirnov property Q1 is satisfied. Hence, $(K, \stackrel{\text { conn }}{\delta})$ is a proximity space.

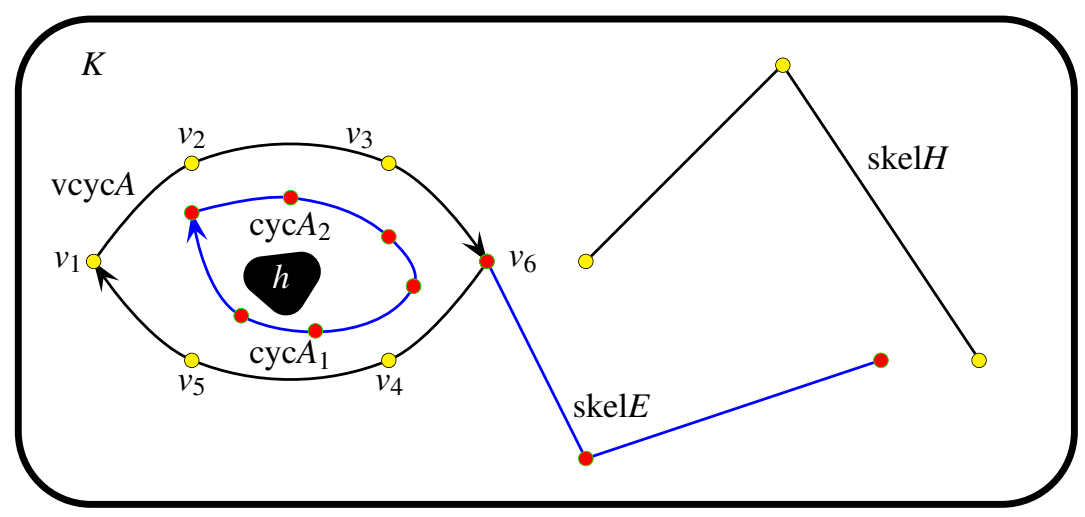

Figure 3: Collection of Skeletons, including a Vortex Cycle with a Hole

\section{Example 3. Connectedness Proximity Space.}

Let $K$ be a collection of skeletons represented in Fig. 3, equipped with the proximity ${ }^{c o n n}$. A pair of skeletons in $K$ are close, provided the skeletons have at least one vertex in common. For example, vortex cycle vcycA and skeleton skelE have vertex $v_{6}$ in common. Hence, from axiom P4conn, we have

$$
v_{6} \in v c y c A \cap \operatorname{skelE} \Leftrightarrow \operatorname{vcycA} \stackrel{\operatorname{conn}}{\delta} \text { skelE }
$$


Skeletons that are not close have no vertices in common. For example, in Fig. 3,

$$
\text { skelE } \stackrel{\text { conn }}{\varnothing} \text { skelH, }
$$

since the pair of skeletons skelE, skelH have no vertices in common.

Theorem 5. Let $K$ be a collection of vortex cycles in a planar cell complex. The space $K$ equipped with the relation $\stackrel{\text { conn }}{\delta}$ is a proximity space.

Proof. A vortex cycle is a collection of concentric 1-cycles. Each 1-cycle is a skeleton. Then vortex cycle is a collection of skeletons and each collection of vortex cycles is also a collection of skeletons. Hence, from Lemma $3, K$ is a connectedness proximity space.

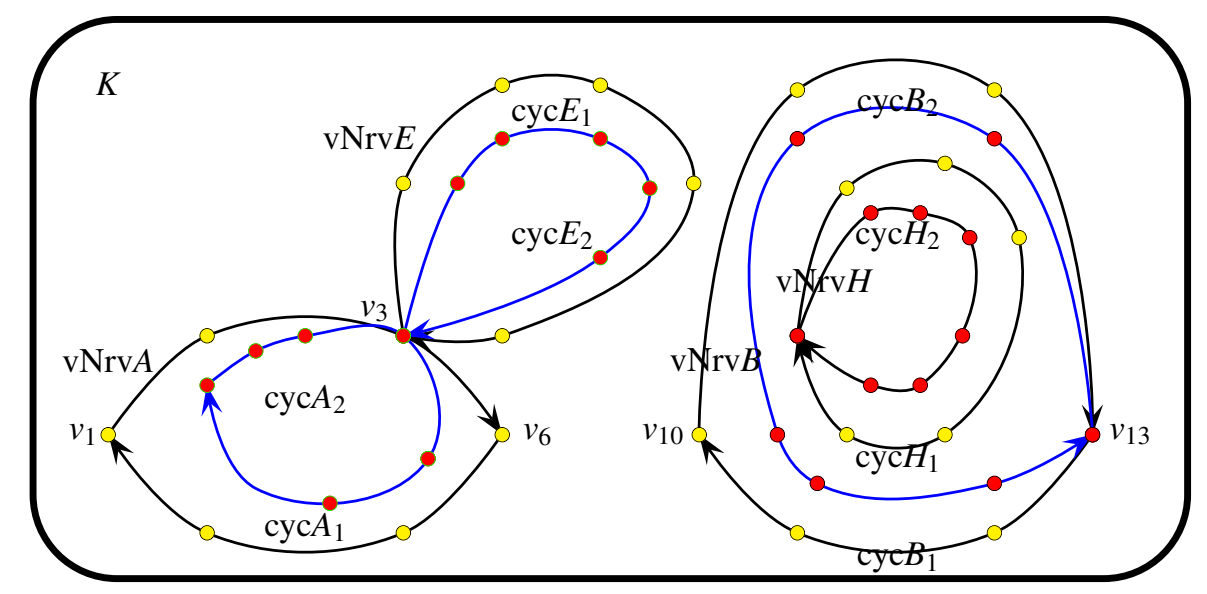

Figure 4: Collection of Proximal Vortex Nerves

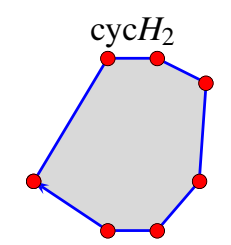

Figure 5: $\mathrm{cycH}_{2} \in \mathrm{vNrvH} \in \mathrm{vNrv} B$ in Fig. 4

\subsection{Vortex Nerves Proximity space}

A vortex cycle $\operatorname{vcyc} A$ containing 1-cycles with a common vertex is an example of a vortex nerve (denoted by $v N r v A$ ). A collection of vortex nerves equipped with the $\delta$ proximity is a connectedness proximity space.

Theorem 6. Let $K$ be a collection of vortex nerves in a planar cell complex. The space $K$ equipped with the relation $\delta$ is a proximity space.

Proof. Each vortex nerve is a collection of intersecting 1-cycles, which are skeletons. The results follows from Lemma 3, since $K$ is also a collection of skeletons equipped with the proximity $\delta$. $\delta$.

\section{Example 4. Vortex Nerves Proximity Space.}

Three vortex nerves $v N r v A, v N r v E$ attached to $v N r v A, v N r v B, v N r v H$ in the interior of $v N r v B$ in a cell complex $K$ are represented in Fig. 4. The filled interior of a 1-cycle in a vortex that appears in Fig. 4 is represented with a shaded interior in cycH $\mathrm{H}_{2} \in \mathrm{vNrvH} \in v \mathrm{NrvB}$ in Fig. 5 . For simplicity, the filled interiors of the 1-cycles in Fig. 4 are hidden (not shaded). Let the collection of vortex nerves $K$ be equipped with the proximity $\stackrel{c}{\delta}$. Vortex nerves are close, provided the nerves have nonempty intersection. For example, $v N r v A \stackrel{c}{\delta} v N r v E$, i.e., $\delta(v N r v A, v N r v E)=0$. Hence, Smirnov property $Q 2$ is satisfied by $(K, \stackrel{c o n n}{\delta})$. Vortex nerves are far (not close), provided the vortex nerves have empty intersection. For example, $v N r v A \stackrel{\operatorname{conn}}{\delta} v N r v E$, i.e., $\delta(v N r v A, v N r v E)=1$ (Smirnov property Q3). We also have, for example,

$$
\begin{aligned}
\delta(v N r v A, v N r v H) & =1=\delta(v N r v B, v N r v H) \text { non-intersecting nerves are far, } \\
\delta(v N r v H, v N r v E) & =1 \text { and } \delta(v N r v A, v N r v E)=0 \\
& \Leftrightarrow \delta(v N r v H, v N r v E) \geq \delta(v N r v A, v N r v E) .
\end{aligned}
$$

In effect, Smirnov property $Q 1$ is satisfied. Hence, $(K, \stackrel{c o n n}{\delta})$ is a connectedness proximity space. 
Example 5. Spacetime Vortex Nerves Proximity Space.

Spacetime vortex nerves (overlapping vortex cycles) have been observed in recent studies of ground vortex aerodynamics by J.P. Murphy and D.G. MacManus [30] and in the vortex flows of overlapping jet streams in ground proximity by J.M.M. Barata, N. Bernardo, P.J.C.T. Santos and A.R.R. Silva [4] and by A.R.R. Silva, D.F.G. Durão, J.M.M. Barata, P. Santos S. Ribeiro [43]. Physical vortex nerves can be observed in the representation of the contours of overlapping turbulence velocity vortices in, for example, Figure 6 in [43, p. 8] and systems of vortex in Figure 7 in P.R. Spalart, M. Kh. Strelets, A.K. Travin and M.L. Slur [42].

The presence of holes in the interiors of vortex nerves in a cell complex equipped with the proximity $\stackrel{\operatorname{conn}}{\delta}$ gives us the following result.

Corollary 1. Let $K$ be a collection of vortex nerves containing holes in their interiors in a planar cell complex. The space K equipped with the relation $\stackrel{c o n n}{\delta}$ is a proximity space.

Proof. Immediate from Theorem 6, since the relationships between vortex nerves in $K$ are unaffected by the presence of holes in the interiors of the nerves.

Example 6. A pair of disjoint vortex nerves containing holes in their interiors is represented in Fig. 6.

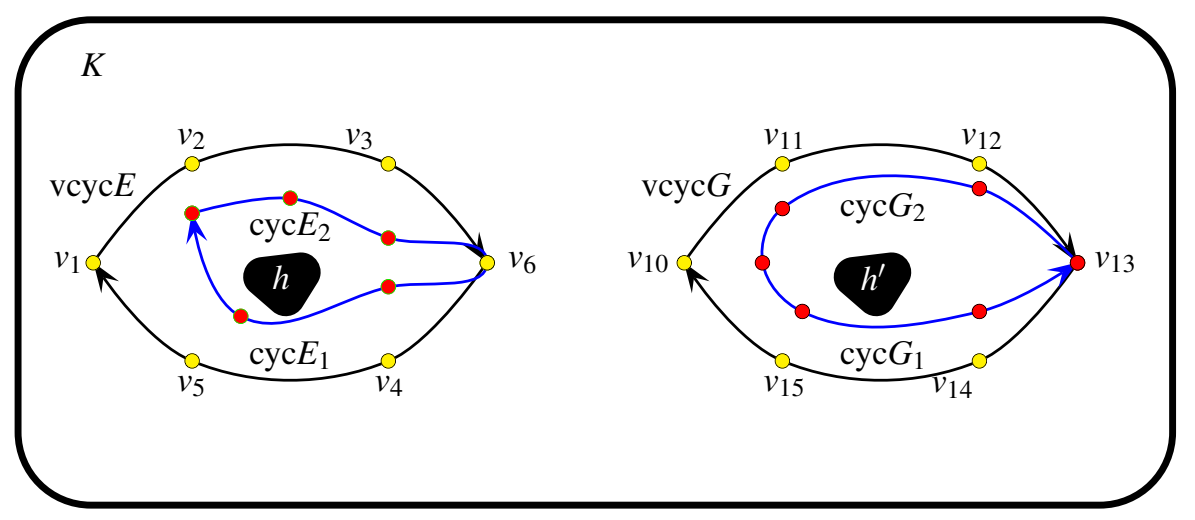

Figure 6: Pair of Disjoint Vortex Nerves With Holes

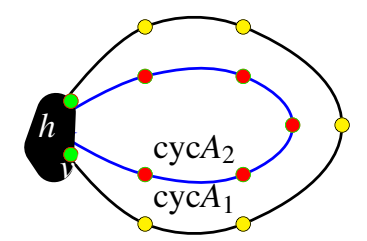

Figure 7: $\left(\operatorname{cyc} A_{1} \cup \operatorname{cyc} A_{2}\right) \cap h$

Problem 3. Let $K$ be a collection of vortex nerves so that the boundary of each of the holes has more than one vertex that is in the intersection 1-cycles in each of the nerves in a planar cell complex. For an example of vortex cycles that overlap vertices on the boundary of a hole, see Fig. 7. Prove that a vortex nerve is destroyed by a hole whose boundary overlaps the nerve cycles in more than one vertex.

Problem 4. Let $K$ be a collection of vortex nerves so that the boundary of each of the holes has a single vertex that is in the intersection of the 1 -cycles in each of the nerves in a planar cell complex. Also let $K$ be equipped the proximity $\delta$. Prove that $K$ is a connectedness proximity space.

\subsection{Neighbourhoods, Set Closure, Boundary, Interior and CW Topology}

The interior of a nonempty set is considered, here. It is the interior of a vortex cycle that leads to strong forms of connectedness proximity on a shapes in cell complex in which the interiors of vortices overlap either spatially or descriptively. Let $A$ be a nonempty set of vertices, $p \in A$ in a bounded region $X$ of the Euclidean plane. An open ball $B_{r}(p)$ with radius $r$ is defined by

$$
B_{r}(p)=\{q \in X:\|p-q\|<r\} .
$$

The closure of $A$ (denoted by $\operatorname{cl} A$ ) is defined by

$$
\operatorname{cl} A=\left\{q \in X: B_{r}(q) \subset A \text { for some } r\right\} \text { (Closure of set } A \text { ). }
$$

The boundary of $A$ (denoted by bdy $A$ ) is defined by

$$
\operatorname{bdy} A=\{q \in X: B(q) \subset A \cap X \backslash A\} \text { (Boundary of set } A \text { ). }
$$


Of great interest in the study of the closeness of vortex cycles is the interior of a shape, found by subtracting the boundary of a shape from its closure. In general, the interior of a nonempty set $A \subset X$ (denoted by $\operatorname{int} A$ ) defined by

$$
\operatorname{int} A=\operatorname{cl} A-\operatorname{bdy} A(\text { Interior of } \operatorname{set} A) \text {. }
$$

Let the cell complex $K$ be a Hausdorff space. Let $A$ be a cell (skeleton) in $K$. Each cell decomposition $A, B \in K$ is called a CW complex, provided

Closure Finiteness Closure of every cell (skeleton) $\mathrm{cl} A$ intersects on a finite number of other cells.

Weak topology $A \in 2^{K}$ is $\operatorname{closed}(A=\operatorname{bdy} A \cup \operatorname{int} A)$, provided $A \cap \operatorname{cl} B$ is closed, i.e.,

$$
A \cap \mathrm{cl} B=\operatorname{bdy}(A \cap \mathrm{cl} B) \cup \operatorname{Int}(A \cap \mathrm{cl} B) \text {. }
$$

$K$ has a topology $\tau$ that is a CW topology [51], [39, $\$ 2.4$, p. 81], provided $\tau$ has the closure finiteness and weak topology properties.

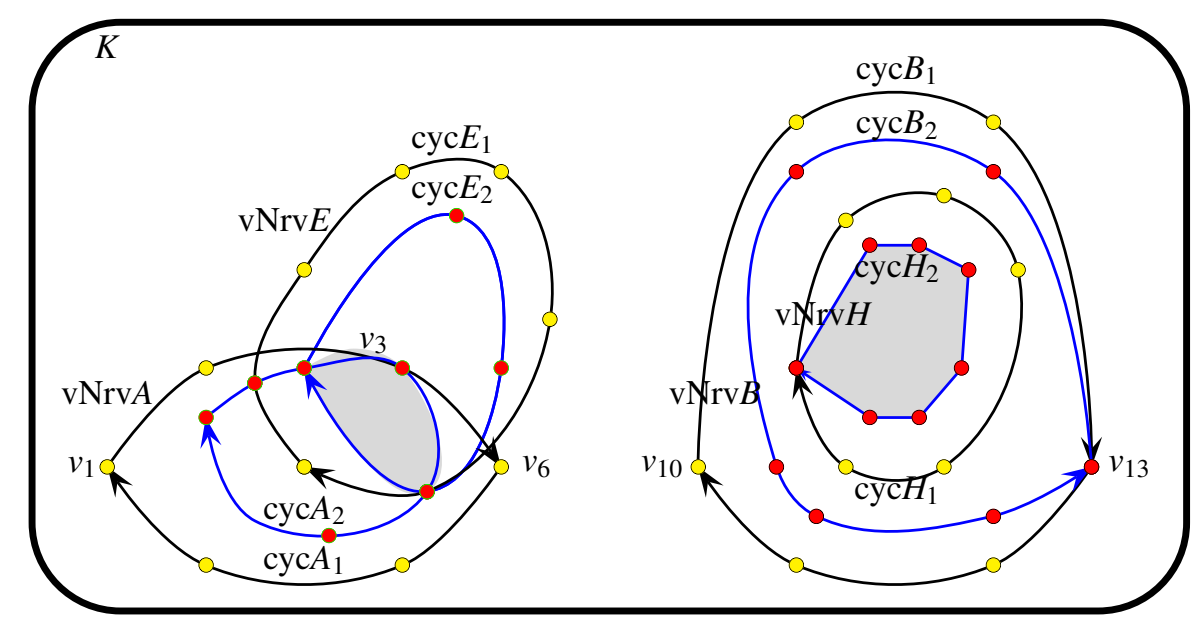

Figure 8: Vortex Nerves with Overlapping Interiors

\subsection{Overlap Connectedness Proximity Space}

In this section, weak and strong connectedness proximities of skeletons arise when we consider pairs of vortex cycles with overlapping

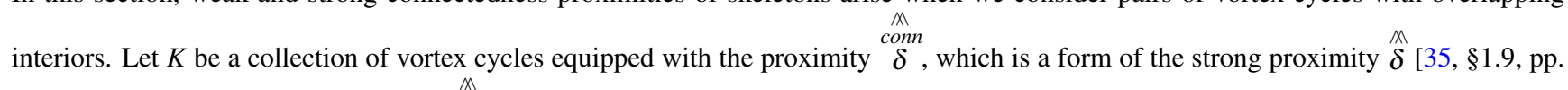
28-30]. The weak and strong forms of $\underset{\text { conn }}{\delta}$ satisfy the following axioms.

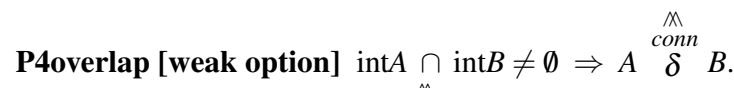

P5overlap [strong option] $A \stackrel{\text { conn }}{\stackrel{\text { }}{\delta} B \Rightarrow A \cap B \neq \emptyset}$

Axiom P4overlap is a rewrite of the C̆ech axiom P4 and axiom P5overlap is addition to the usual C̆ech axioms. It is easy to see that $\hat{\delta}$ satisfies the remaining C̆ech axioms after replacing $\delta$ with $\stackrel{\wedge}{\delta}$. Let $A, B, C \in K$, a cell complex space equipped with the proximity $\begin{gathered}\text { conn } \\ \delta\end{gathered}$, which satisfies the following axioms.

Overlap Connectedness proximity axioms.

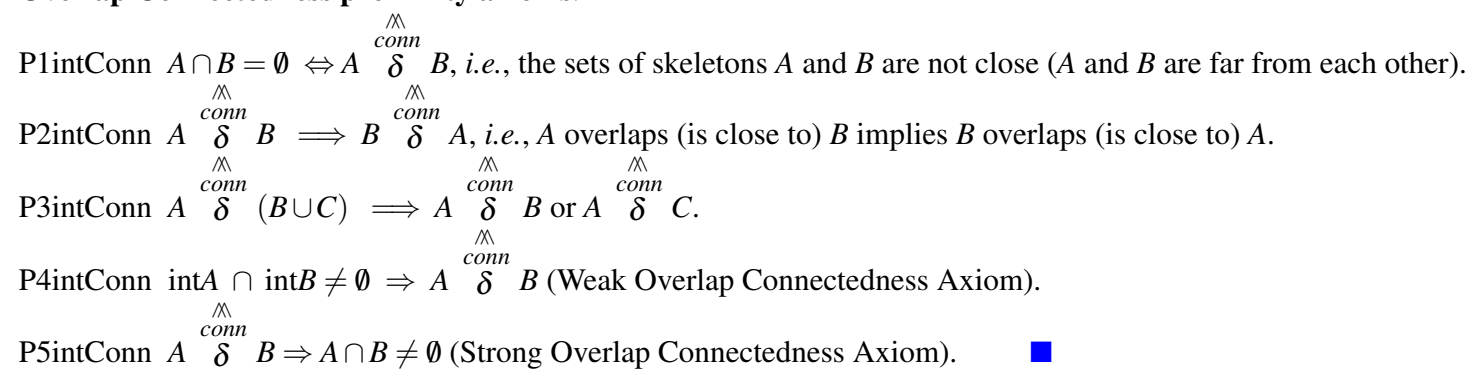


An overlap connectedness space is denoted by $(K, \stackrel{\substack{\text { conn } \\ \delta}}{)}$. Skeletons $A, B$ in $K$ are close, provided the interior int $A$ has nonempty intersection with the interior int $A$

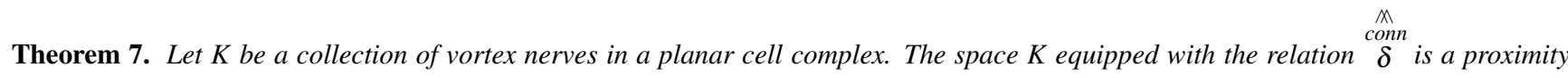
space.

Proof. The result follows from Lemma 3, since $K$ is also a collection of skeletons equipped with the proximity ${ }^{\operatorname{conn}} \delta$.

\section{Example 7. Overlapping Vortex Nerves.}

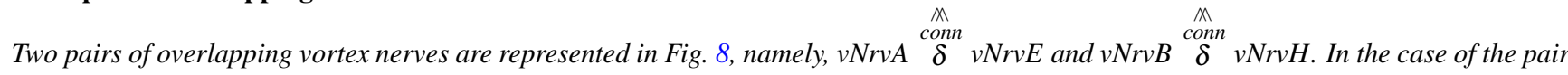
of vortex nerves $v N r v A, v N r v E$, the gray region for these nerves in Fig. 8 represents the nonempty intersection of the interior of the 1-cycle intcyc $A_{2} \in v N r v A$ and the interior of the 1-cycle intcyc $E_{2} \in v N r v E$. From axiom P4intConn, we have

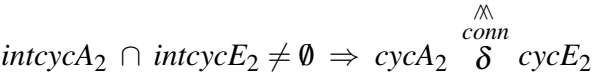

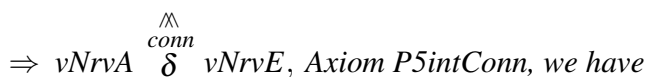

$$
\begin{aligned}
& v N r v A \stackrel{\substack{\text { conn } \\
\delta}}{\delta} v r v E \Rightarrow \operatorname{intcyc} A_{2} \cap \operatorname{intcyc} E_{2} \neq \emptyset .
\end{aligned}
$$

Concentric vortex nerves $v \mathrm{NrvB}, v \mathrm{NrvH}$ are also represented in Fig. 8, The interior Intcyc $\mathrm{H}_{2}$ is represented in Fig. 5in the vortex nerve $v N r v H$, which is in the interior of vortex nerve vNrvB. Again, from axiom P4intConn, we have

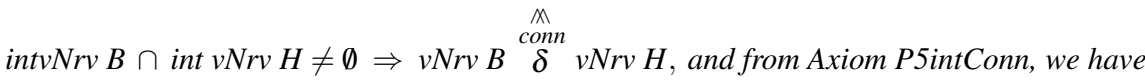

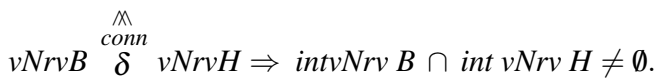

Example 8. Spacetime Vortex Cycles: Overlapping Electromagnetic Vortices.

I.V. Dzedolik observes that an electromagnetic vortex is formed by photons that possess some net angular momentum about the longitudinal axis of a dielectric waveguide [12, p. 135]. Photons are almost massless objects that carry energy from an emitter to an absorber [49].

Modeling spiraling vortices as vortex cycles equipped with the $\stackrel{\text { conn }}{\delta}$ proximity suggests the possibility of obtaining an expanded range of measurements in vortex optics. N.M. Litchinitser observes that vortex-preshaped femtosecond laser pulses indicate the possibility of achieving repeatable and predictable spatial and temporal distribution in using metamaterials in light filamentation [27, p. 1055]. The overlap connectedness proximity space approach to characterizing, analysing and modelling neighboring photons gains strength by considering recent work by $M$. Hance on isolating and comparing different forms of photons (and photon vortical flux) [21, \$4, pp. 8-11].

\subsection{Descriptive Connectedness Proximity}

In this section, weak and strong descriptive connectedness proximities of skeletons arise when we consider pairs of vortex cycles with matching description. A vortex cycle description is a feature vector that contains features values extracted from vortices with what are $\stackrel{\text { An }}{\text { conn }}$

known as probe functions. Let $K$ be a collection of vortex cycles equipped with the descriptive proximity $\delta_{\Phi}$, which is an extension of the descriptive proximity $\hat{\delta}_{\Phi}\left[7, \S 3-4\right.$, pp. 95-98]. The mapping $\Phi: K \longrightarrow \mathbb{R}^{n}$ yields an $n$-dimensional feature vector in Euclidean space $\mathbb{R}^{n}$ either a vortex cyc $A \in K$ (denoted by $\Phi(\operatorname{cyc} A)$ ) or a vortex cycle vcyc $E$ in $K$ (denoted by $\Phi(\operatorname{vcyc} E)$ ) or a vortex nerve vNrv $H$ in $K$ (denoted by $\Phi(\mathrm{vNrvH})$ ). For the axioms for a descriptive proximity, the usual set intersection is replaced by descriptive intersection [34, $\S 3$ ] (denoted by $\underset{\Phi}{\bigcap}$ ) defined by

$$
\underset{\Phi}{A \cap B}=\{x \in A \cup B: \Phi(x) \in \Phi(A), \Phi(x) \in \Phi(B)\} .
$$

The descriptive closure of $A$ (denoted by $\operatorname{cl}_{\Phi} A$ ) $[35, \S 1.4$, p. 16] is defined by

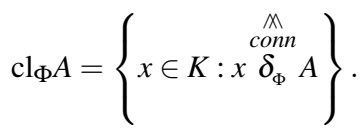

$$
\stackrel{\text { M }}{\text { conn }}
$$

The weak and strong forms of $\delta_{\Phi}^{c o n n}$ satisfy the following axioms. 


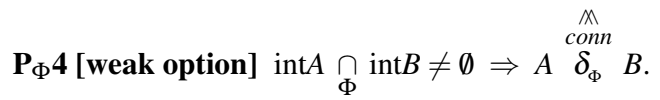

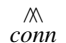

$\mathbf{P}_{\Phi} 5$ option] $A \stackrel{c}{\delta_{\Phi}} B \Rightarrow \underset{\Phi}{A \cap} B \neq \emptyset$

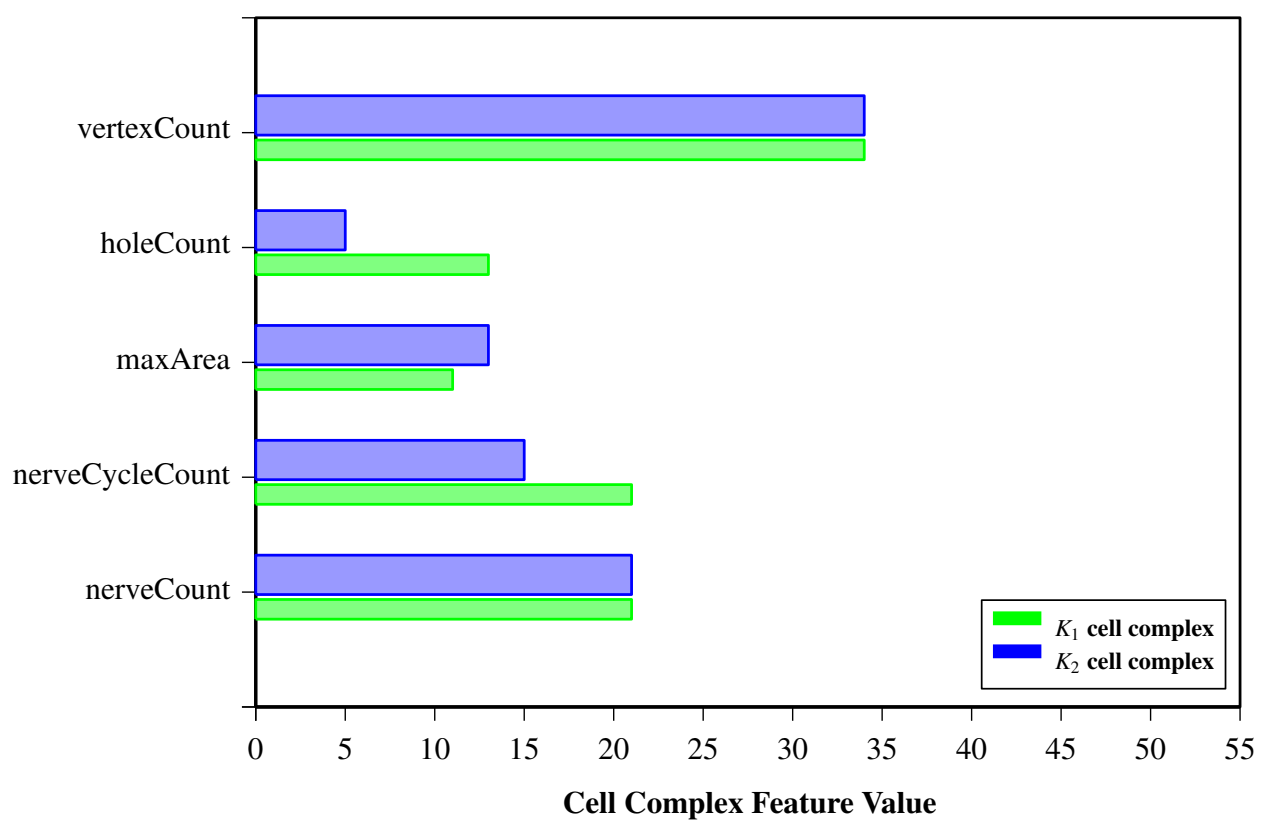

Figure 9: Comparison of Cell Complex Feature Values

Axiom $\mathbf{P}_{\Phi} \mathbf{4}$ is a rewrite of the C̆ech axiom $\mathbf{P} 4$ and axiom $\mathbf{P}_{\Phi} \mathbf{5}$ is an addition to the usual C̆ech axioms. It is easy to see that $\begin{gathered}\text { conn } \\ \delta_{\Phi}\end{gathered}$ satisfies the

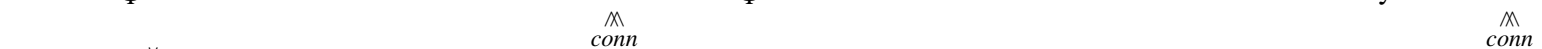
remaining C̆ech axioms after replacing $\delta$ with $\stackrel{c o n n}{\delta}$. Let $A, B, C \in K$, a cell complex space equipped with the proximity ${ }^{c o n n}$, which satisfies the following axioms.

\section{Descriptive Overlap Connectedness proximity axioms.}

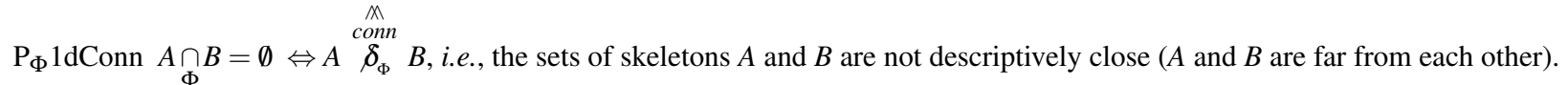

$\mathrm{P}_{\Phi} 2 \mathrm{dConn} A \stackrel{\substack{\text { conn } \\ \delta_{\Phi}}}{\longrightarrow} B \stackrel{\text { conn }}{\delta_{\Phi}} A$, i.e., $A$ is descriptively close to $B$ implies $B$ is descriptively close to $A$.

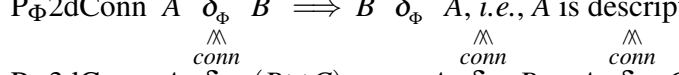

$\mathrm{P}_{\Phi} 3 \mathrm{~d}$ Conn $A \stackrel{\text { conn }}{\delta_{\Phi}}(B \cup C) \Longrightarrow A \stackrel{\text { conn }}{\delta_{\Phi}} B$ or $A \stackrel{\text { conn }}{\delta_{\Phi}} C$

$\mathrm{P}_{\Phi} 4 \mathrm{dConn} \operatorname{int} A \underset{\Phi}{\cap} \operatorname{int} B \neq \emptyset \Rightarrow A \stackrel{\text { conn }}{\delta_{\Phi}} B$ (Weak Descriptive Connectedness Axiom).

$\mathrm{P}_{\Phi} 5 \mathrm{dConn} A \stackrel{\substack{\text { conn } \\ \delta_{\Phi}}}{ } B \Rightarrow A \underset{\Phi}{\cap} B \neq \emptyset$ (Strong Descriptive Connectedness Axiom).

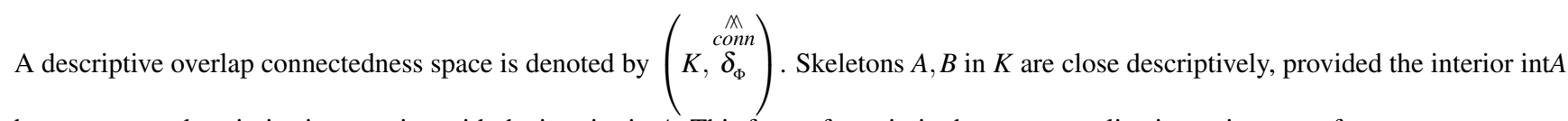
has nonempty descriptive intersection with the interior int $A$. This form of proximity has many applications, since we often want to compare objects such as 1-cycles by themselves or vortex cycles or the more complex vortex nerves that do not overlap spatially or at the same time.

Example 9. Descriptive Connectedness Overlap of Disjoint Vortex Cycles in Spacetime.

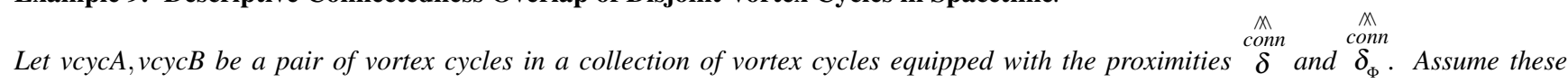




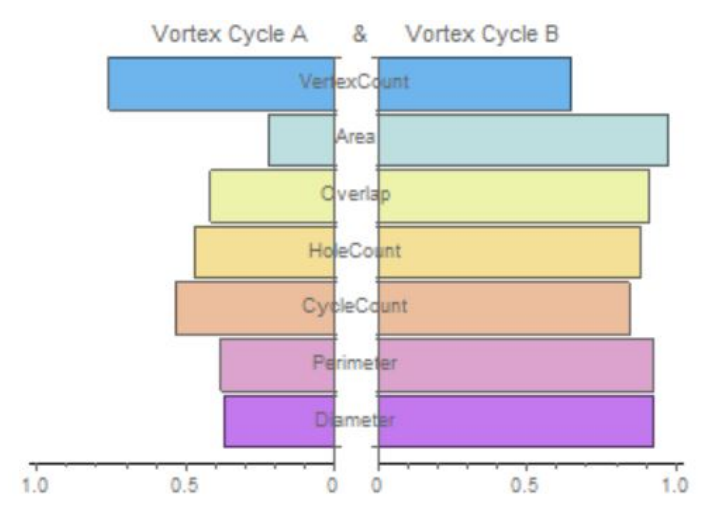

Figure 10: Comparison of Vortex Cell Feature Values

vortices represent non-overlapping electromagnetic vortexes that have matching descriptions in spacetime, e.g., $\Phi(v c y c A)=\Phi(v c y c B)=$

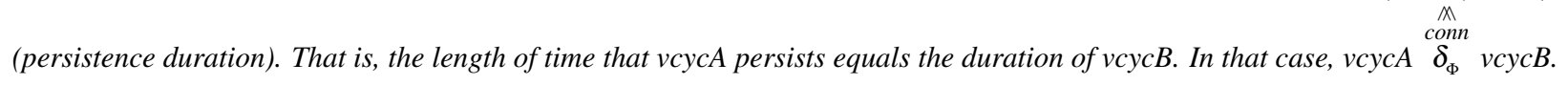

Example 10. Descriptive Connectedness Overlap of Cell Complexes.

The bar graph ${ }^{2}$ in Fig. 9 compares feature values for a pair of cell complexes, namely, vertex count, hole count, maximum vortex cycle area, nerve cycle count and nerve count. From the bar graph, $K_{1} \stackrel{\substack{c \\ \delta_{\Phi}}}{\delta_{\Phi}} K_{2}$, since

$\Phi\left(K_{1}\right.$ vertexCount $)=\Phi\left(K_{2}\right.$ vertexCount $)=35$, and

$\Phi\left(K_{1}\right.$ nerveCount $)=\Phi\left(K_{2}\right.$ nerveCount $)=21$.

This is the case, even though the hole count and nerve cycle count are far apart.

Example 11. Absence of Descriptive Connectedness of Sample Vortex Cycles.

The bar graph in Fig. 10 compares normalized feature values for a pair of sample vortex cycles vcycA, vcycB, namely, vertex count, vortex cycle area, overlap (i.e., number of overlapping 1-cycles in a vortex cycle), hole count, cycle count, perimeter (i.e., length of the boundary of a vortex cycle), diameter (i.e., maximum distance between a pair of vertices on the boundary of a vortex cycle). From the bar graph, it is apparent that vcycA $\stackrel{\substack{c o n n \\ \delta_{\Phi}}}{ } v c y c B$, since there are no matching feature values for the sample pair of vortex cycles.

Theorem 8. Let $K$ be a collection of vortex cycles in a planar cell complex. The space $K$ equipped with the relation ${ }_{\Phi} \delta_{\Phi}$ is a proximity space.

Proof. The result follows from Lemma 3, since each vortex cycle in $K$ is also a collection of skeletons equipped with the proximity $\delta_{\Phi}$.

Corollary 2. Let $K$ be a collection of vortex nerves in a planar cell complex. The space $K$ equipped with the relation $\delta_{\Phi}$ is a proximity space.

Proof. The result follows from Theorem 8 , since each vortex nerve in $K$ is also a collection of intersecting vortex cycles equipped with the proximity $\stackrel{\substack{\text { conn } \\ \delta_{\Phi}}}{.}$

Example 12. Non-Overlapping Vortex Nerve with Matching Descriptions.

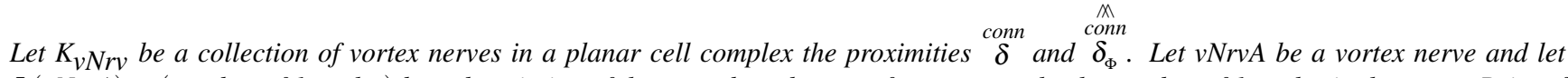
$\Phi(v N r v A)=($ number of 1-cycles $)$ be a description of the nerve based on one feature, namely, the number of 1-cycles in the nerve. Pairs of

${ }^{2}$ Many thanks to M.Z. Ahmad for the $\mathrm{LT}_{\mathrm{E}} \mathrm{X}$ script used to display this bar graph, which does not depend on an external file. 
non-overlapping vortex nerves with matching descriptions are represented in Fig. 8, namely,

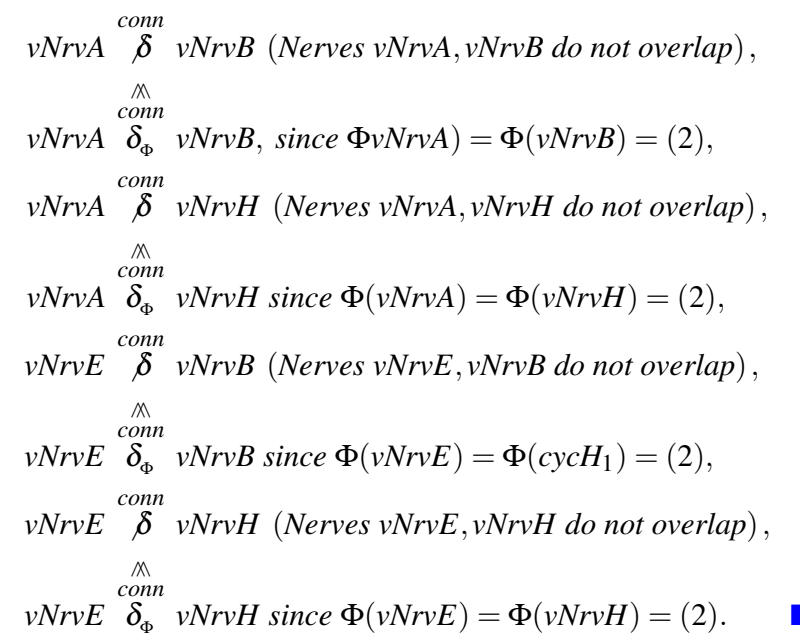

\section{Example 13. Non-Overlapping Vortex Nerve Cycles with Matching Descriptions.}

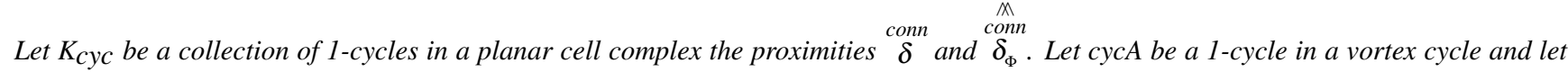
$\Phi($ cycA $)=$ (number of vertices) be a description of the cycle based on one feature, namely, the number of vertices in the cycle. Pairs of non-overlapping vortex nerves containing 1-cycles with matching descriptions are represented in Fig. 8, namely,

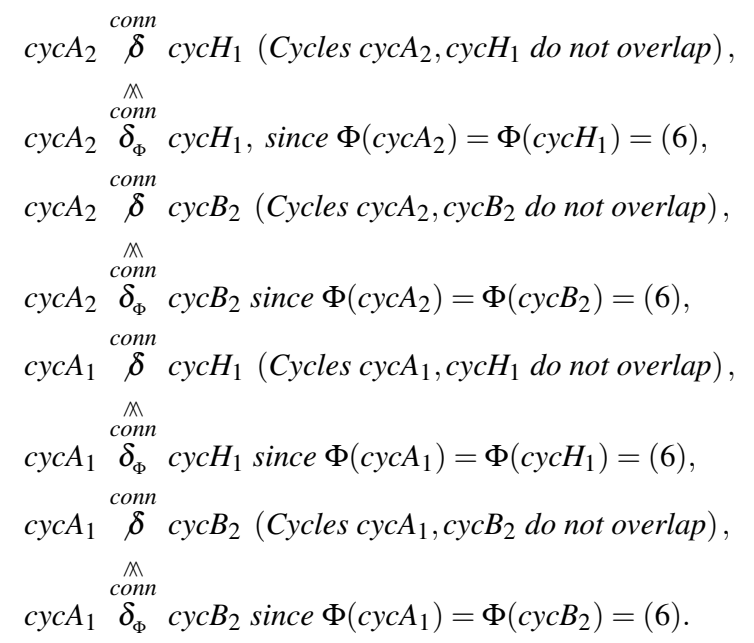

\subsection{Vortex Cycle Spaces Equipped with Proximal Relators}

This section introduces a connectedness proximal relator [36] (denoted by $\mathscr{R}$ ), an extension of a Száz relator [45], which is a non-void collection of connectedness proximity relations on a nonempty cell complex $K$. A space equipped with a proximal relator $\mathscr{R}$ is called a proximal relator space (denoted by $(K, \mathscr{R})$ ).

Example 14. Proximal Relator Space. Example 12 introduces a proximal relator space $\left(K_{v N r v},\left\{\begin{array}{c}\text { conn conn } \\ \delta\end{array}, \delta_{\Phi}^{x}\right\}\right)$, useful in measuring, comparing, and classifying collections of vortex nerves that either have or do not have matching descriptions. Similarly, Example 13 introduces a proximal relator $\left(K_{c y c},\left\{\begin{array}{c}\text { conn conn } \\ \delta, \\ \delta_{\Phi}\end{array}\right\}\right)$, useful in the study of collections of 1 -cycles that either have or do not have matching descriptions.

The connection between $\stackrel{\wedge}{\delta}$ and $\delta$ is summarized in Lemma 4.

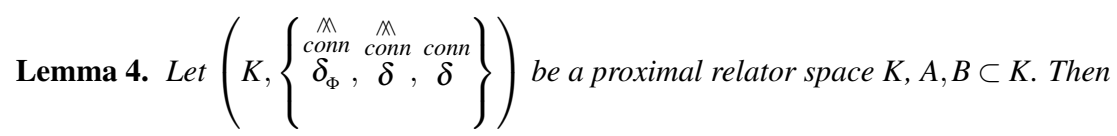

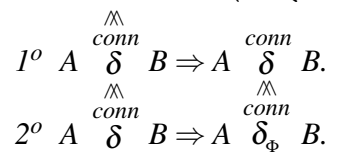


Proof.

$1^{o}$ : From Axiom P5conn, $A \stackrel{\substack{c o n \\ \delta}}{\operatorname{con}} B$ implies $A \cap B \neq \emptyset$, which implies $A \stackrel{\text { conn }}{\delta} B$. From Lemma $2, A \stackrel{\text { conn }}{\delta} B$ implies $A \cap B \neq \emptyset$, which implies $A \delta B$ (from C̆ech Axiom P4).

$2^{o}:$ From (1), there are cyc $x \in A$, cyc $y \in B$ common to $A$ and $B$. Hence, $\Phi(\operatorname{cyc} x)=\Phi(\operatorname{cyc} y)$, which implies $A \underset{\Phi}{\bigcap} B \neq \emptyset$. Then, from the descriptive connectedness Axiom $P_{\Phi} 4 \operatorname{conn}, A \underset{\Phi}{\bigcap} B \neq \emptyset \Rightarrow A \stackrel{\text { conn }}{\delta_{\Phi}} B$. This gives the desired result.

Let vNrvA be a vortex nerve. By definition, vNrvA is collection of 1-cycles with nonempty intersection. The boundary of vNrvA (denoted by bdyvNrvA) is a sequence of connected vertices. That is, for each pair of vertices $v, v^{\prime} \in \operatorname{bdyvNrv} A$, there is a sequence of edges, starting with vertex $v$ and ending with vertex $v^{\prime}$. There are no loops in bdyvNrvA. Consequently, bdyvNrvA defines a simple, closed polygonal curve. The interior of bdyvNrvA is nonempty, since $\operatorname{Nrv} A$ is a collection of filled polytopes. Hence, by definition, a vNrvA is also a nerve shape.

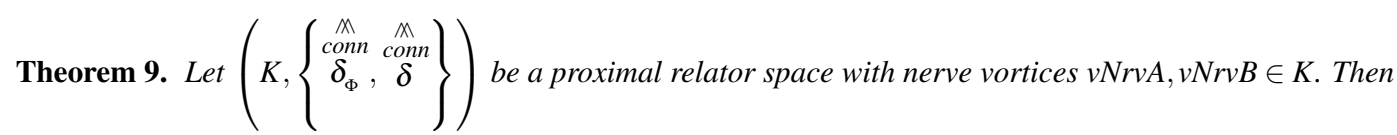

$1^{o} v N r v A \stackrel{\substack{\wedge \\ \text { conn }}}{\delta} v N r v B$ implies $v N r v A \stackrel{\substack{\text { conn } \\ \delta_{\Phi}}}{\delta_{N}}$ NrvB.

$2^{\circ}$ A 1-cycle cycE $\in v N r v A \cap v N r v B$ implies cycE $\in v N r v A \bigcap_{\Phi} v N r v B$.

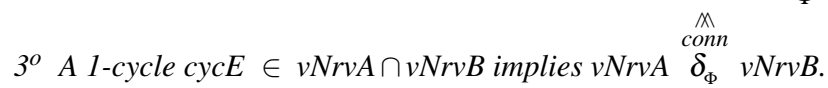

Proof.

$1^{o}$ : Immediate from part (2) of Lemma 4.

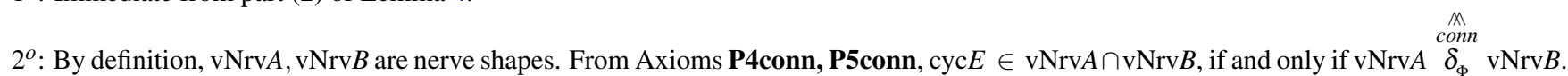
Consequently, $\operatorname{cyc} E$ is common to $\mathrm{vNrv} A, \mathrm{vNrv} B$. Then there is a cycle $\operatorname{cyc} E \in \operatorname{Nrv} A$ with the same description as a $\operatorname{cycle} \operatorname{cyc} E \in \mathrm{vNrv} B$. Let $\Phi(\operatorname{cyc} E)$ be a description of $\operatorname{cyc} E$. Then, $\Phi(\operatorname{cyc} E) \in \Phi(\mathrm{vNrv} A) \& \in \Phi(\operatorname{cyc} E) \in \Phi(\mathrm{vNrv} B)$, since cyc $E \in \operatorname{vNrv} A \cap \mathrm{vNrv} B$. Hence, $\operatorname{cyc} E \in \operatorname{vNrv} A \underset{\Phi}{\bigcap} \mathrm{vNrv} B$.

$3^{o}$ : Immediate from (2) and Lemma 4.

\section{Main Results}

This section gives some main results for collections of proximal vortex cycles and proximal vortex nerves.

\subsection{Topology on Vortex Cycle Spaces}

This section introduces the construction of topology (homology) classes of vortex cycles and vortex nerves. Topology classes have proved to be useful in classifying physical objects such as quasi-crystals [11] and in knowledge extraction [17]. Such classes provide a basis for knowledge extraction about proximal vortex cycles and nerves. A strong beneficial side-effect of the construction of such classes is the ease with which the persistence of homology class objects can be computed (see, e.g., [16], [2]). More importantly, the construction of topology classes leads to problem size reduction (see, e.g., [31, §3.1, p. 5]).

Lemma 5. Let $K$ be a nonempty collection of finite skeletons on a finite cell complex $K$ that is a Hausdorff space equipped the proximity $\stackrel{c o n n}{\delta}$. From the pair $(K, \stackrel{c o n n}{\delta})$, a Whitehead Closure Finite Weak $(C W)$ Topology can be constructed.

Proof.

From Lemma $3,(K, \stackrel{c o n n}{\delta})$ is a connectedness proximity space. Let $\mathrm{sk} A, \mathrm{sk} B$ be skeletons in a finite cell $\operatorname{complex} K$. The closure cl(sk $A)$ is finite and includes the connected vertices on the boundary $\operatorname{bdy}(\operatorname{sk} A)$ and in the interior $\operatorname{bdy}(\operatorname{sk} A)$ of $\operatorname{sk} A$. Since $K$ is finite, $\operatorname{cl}(\operatorname{sk} A)$ intersects a only a finite number of other skeletons in $K$. The intersection $\operatorname{sk} A \cap \operatorname{sk} B \neq \emptyset$ is itself a finite skeleton, which can be either a single vertex or a set of edges common to $\operatorname{sk} A, \operatorname{sk} B$. In that case, $\operatorname{sk} A \underset{c o n n}{\delta} \operatorname{sk} B$. By definition, $\operatorname{sk} A \cap \operatorname{sk} B$ is a skeleton in $K$. Consequently, whenever $\mathrm{sk} A \stackrel{\operatorname{conn}}{\delta} \mathrm{sk} B$, then $\operatorname{sk} A \cap \mathrm{sk} B \in K$. Hence, $(K, \stackrel{c o n n}{\delta})$ defines a Whitehead CW topology.

Theorem 10. Let $K$ be a nonempty collection of finite skeletons on a finite cell complex $K$ that is a Hausdorff space equipped the proximity

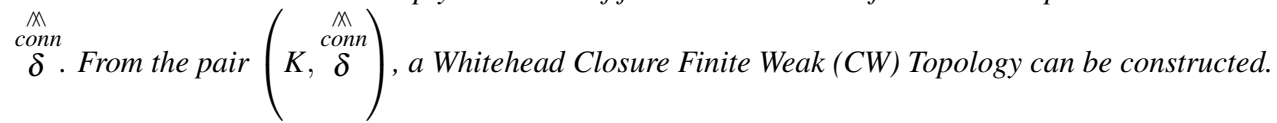

Proof.

Immediate from Lemma 5.

Next, we construct a Leader uniform topology on a collection of vortex cycles equipped with the descriptive connectedness proximity $\delta_{\Phi}^{c o n n}$. 
Definition 1. Let $X$ be a nonempty set. For each given set $A \in 2^{X}$, form a cluster containing all subsets $B \in 2^{X}$ such that $A \cap B \neq \emptyset$. The intersection as well as the union of clusters belong to $K$, defining a Leader uniform topology on $K$, namely, the collection of all uniform clusters on $K$.

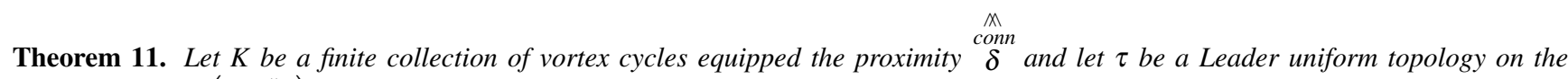
proximity space $\left(K, \begin{array}{c}\text { conn } \\ \delta\end{array}\right)$. Then each cluster of vortex cycles $E \in \tau$ has a $C W$ topology on $E$.

Proof.

Ponn

Each $E \in \tau$ is a finite collection of vortex cycles equipped with the proximity ${ }_{\delta}^{c o n n}$. Each closure $\operatorname{cl}(\operatorname{vcyc} H) \in E$ intersects with a finite number of other vortex cycles in $E$, since $E$ is finite (closure finiteness property). Let $\operatorname{cl}(\operatorname{vcyc} A), \operatorname{cl}(\operatorname{vcyc} B) \in E$. For int $(\operatorname{vcyc} A) \cap$ $\operatorname{int}(\operatorname{vcyc} B) \neq \emptyset \Rightarrow \operatorname{cl}(\operatorname{vcyc} A) \stackrel{\substack{\text { conn } \\ \delta}}{d} \mathrm{cl}(\operatorname{vcyc} B)$, from Axiom P4intConn (weak topology property). Hence, $E$ has a CW topology.

For descriptive proximity spaces, the construction of Leader uniform topologies is accomplished by considering the descriptive intersection $\underset{\Phi}{\bigcap}$ and descriptive union $\underset{\Phi}{\bigcup}$ of nonempty sets of vortex cycles. Let $K$ be a nonempty collection of vortex cycles, $A, B \in K$. Then descriptive union $\underset{\Phi}{\cup}$ is defined by

$A \underset{\Phi}{\cup} B=\{E \in K: \Phi(E) \in \Phi(A \cup B)\}$ (Descriptive union of sets of vortex cycles).

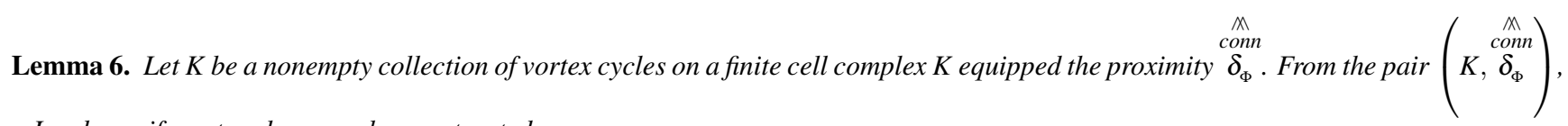
a Leader uniform topology can be constructed.

Proof.

We have $\Phi(K)=\{\Phi(\operatorname{vcyc} A)$ : vcyc $A \in K\}$, the feature space for $K$. Let $\operatorname{vcyc} A \underset{\Phi}{\cap} \mathrm{vcyc} B \neq \emptyset$ be descriptive intersection of a pair of vortex $\stackrel{x}{\text { conn }}$

cycles vcyc $A, \mathrm{vcyc} B$ in $K$. From Axiom $P_{\Phi} 4 \operatorname{conn}, \mathrm{vcyc} A \stackrel{\operatorname{conn}}{\delta_{\Phi}} \mathrm{vcyc} B$. For each given vcyc $A$, find all vortex cycles vcyc $B \in K$ with nonempty

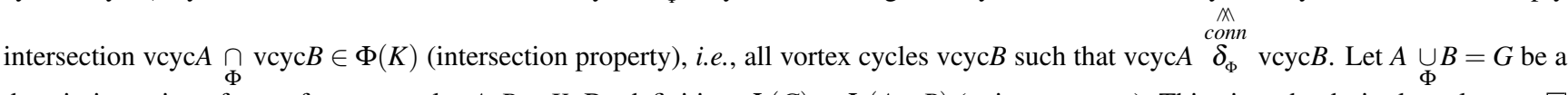
descriptive union of sets of vortex cycles $A, B \in K$. By definition, $\Phi(G) \in \Phi(A \cup B)$ (union property). This gives the desired result.

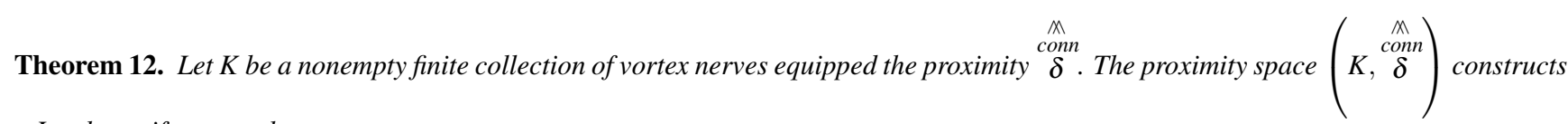
a Leader uniform topology.

Proof.

Immediate from Lemma 6.

From what we have observed so far, a form of problem reduction results from the construction of CW topology on a cluster in a Leader uniform topology.

Theorem 13. Let $\mathscr{C}$ be a Leader uniform topology cluster in a collection of skeletons $K$ equipped the proximity $\delta$. The proximity space $(\mathscr{C}, \stackrel{c o n n}{\delta})$ constructs a CW topology.

Proof.

Immediate from Lemma 5.

Corollary 3. Let $\mathscr{C}$ be a Leader uniform topology cluster in a collection of skeletons $K$ equipped the proximity $\begin{gathered}\text { conn } \\ \delta\end{gathered}$ The proximity space

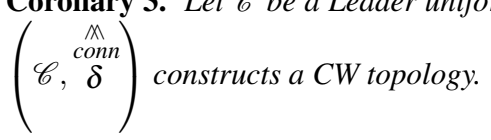

Proof.

Immediate from Theorem 13.

Corollary 4. Let $\mathscr{C}$ be a Leader uniform topology cluster in a collection of vortex cycles $K$ equipped the proximity $\begin{gathered}\text { conn } \\ \delta\end{gathered}$ The proximity $\operatorname{space}(\mathscr{C}, \stackrel{\substack{\text { conn } \\ \delta}}{\delta})$ constructs a CW topology.

Proof.

Immediate from Theorem 13. 
Corollary 5. Let $\mathscr{C}$ be a Leader uniform topology cluster in a collection of vortex nerves $K$ equipped the proximity ${ }^{\wedge}{ }^{\wedge}$. The proximity space $\left(\begin{array}{c}\text { M } \\ \text { conn } \\ \delta\end{array}\right)$ constructs a CW topology.

Proof.

Immediate from Theorem 13.

\subsection{Homotopic Types of Vortex Cycles and Vortex Nerves}

Theorem 14. [15, §III.2, p. 59] Let $\mathscr{F}$ be a finite collection of closed, convex sets in Euclidean space. Then the nerve of $\mathscr{F}$ and the union of the sets in $\mathscr{F}$ have the same homotopy type.

Lemma 7. Let cycA be a vortex cycle in a finite collection of closed, convex skeletons in a cell complex K. Then vortex cycle cycA and the union of the skeletons in cycA have the same homotopy type.

Proof. From Theorem 14, we have that the union of the skeletons sk $E \in \operatorname{cyc} A$ and $\operatorname{cyc} A$ have the same homotopy type.

Theorem 15. Let $K$ be a finite collection of vortex cycles equipped the proximity $\stackrel{M}{\text { conn }}$ and let $\tau$ be a Leader uniform topology on the proximity space $(K, \stackrel{\substack{c o n n \\ \delta}}{ })$. Then each cluster of closed, convex vortex cycles $\mathscr{C} \in \tau$ and the union of vortex cycles in $\mathscr{C}$ have the same homotopy type.

Proof. Each vortex cycle vcyc $A$ in $\mathscr{C}$ is constructed from a collection of closed, convex skeletons in the cell complex $K$. Consequently, $\mathscr{C}$ is a collection of closed, convex vortex cycles. Hence, from Lemma 7, we have that the union of the vortex cycles cyc $A \in \mathscr{C}$ and $\mathscr{C}$ have the same homotopy type.

Corollary 6. Let $K$ be a finite collection of vortex nerves equipped the proximity $\stackrel{\text { conn }}{\delta}$ and let $\tau$ be a Leader uniform topology on the proximity space $(K, \stackrel{c o n n}{\delta})$. Then each cluster of closed, convex vortex nerves $\mathscr{N} \in \tau$ and the union of vortex nerves in $\mathscr{N}$ have the same homotopy type.

Proof.

Immediate from Theorem 15, since vortex nerve is a collection of intersecting closed convex vortex cycles in $K$.

\subsection{Open Problems}

This section identifies open problems emerging from the study of proximal vortex cycles and proximal vortex nerves. Vortex cycles can either be spatially close (overlapping vortex cycles have one or more common vertices) or descriptively close (pairs of vortex cycles that intersect descriptively). For such cell complexes, we have the following open problems.

open- $1^{o}$ Vortex photons can be spatially close (overlap). From Theorem 11, a CW topology can be constructed on each cluster of vortex photons in a uniform Leader topology on a collection of vortex photons. In that case, the problem of considering the spatial closeness of vortex photons for classification and analysis purposes, is simplified by considering a CW topology on each cluster of intersecting vortex photons. This is a form of problem reduction, which has not yet been attempted.

open- $2^{o}$ The space between the spiraling flux of vortex photons can be viewed as holes. Modelling vortex photons with holes using a combination of connectedness proximity and CW topology on clusters of such photons for classification and analysis purposes, is an open problem. This is a form of knowledge extraction.

open- $3^{o}$ It is well-known that real elementary particles can have the form of knots [18], which have various forms in knot theory [46]. Vortex cycles can be viewed as collections of intersecting knots. The collection of all possible configurations of spatially close vortex cycles is an open problem.

open- $4^{o}$ A class of elementary particles known as glueballs exist as knotted chromodynamics flux lines [18]. Vortex nerves can be viewed as collections of intersecting (overlapping) glueballs. The collection of all possible configurations of spatially close vortex nerves is an open problem.

open- $5^{\circ}$ From what has been observed in this paper, vortex cycles can be spatially close (overlap) vortex nerves. The collection of all possible configurations of vortex cycles spatially close to vortex nerves is an open problem.

open- $6^{o}$ Let the cell complex $K$ be a Hausdorff space equipped with ${ }_{\delta_{\Phi}}$ and descriptive closure cl ${ }_{\Phi}$. Let $A$ be a cell (skeleton) in $K$. A descriptive $\mathrm{CW}$ complex can be defined on each cell decomposition $A, B \in K$, if and only if

descriptive Closure Finiteness Closure of every cell (skeleton) $\mathrm{cl}_{\Phi} A$ intersects on a finite number of other cells. descriptive Weak topology $A \in 2^{K}$ is descriptively closed $\left(A=\operatorname{cl}_{\Phi} A\right)$, provided $A \underset{\Phi}{\bigcap_{\Phi}} \operatorname{cl}_{\Phi} B$ is closed, i.e., $A \underset{\Phi}{A} \operatorname{cl} B=\operatorname{cl} l_{\Phi}(A \cap \operatorname{cl} B)$.

Prove that $K$ has a topology $\tau$ that is a descriptive CW topology, provided $\tau$ has the descriptive closure finiteness and descriptive weak topology properties. 


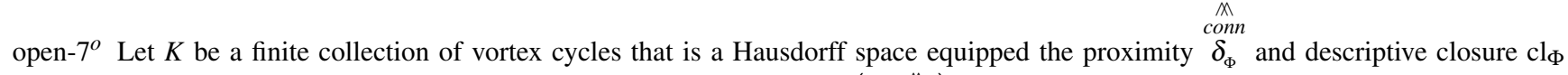
and let $\tau$ be a Leader uniform topology on the proximity space $\left(K, \begin{array}{c}\text { conn } \\ \delta_{\Phi}\end{array}\right)$. Prove that each cluster of vortex cycles $E \in \tau$ has a descriptive $\mathrm{CW}$ topology on $E$.

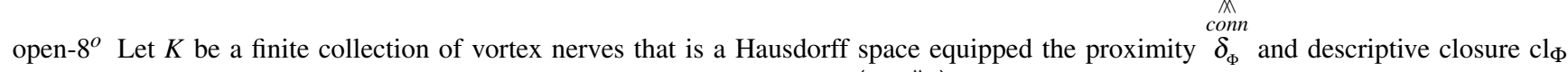
and let $\tau$ be a Leader uniform topology on the proximity space $\left(K, \begin{array}{c}\text { conn } \\ \delta_{\Phi}\end{array}\right)$. Prove that each cluster of vortex cycles $E \in \tau$ has a descriptive CW topology on $E$.

open- $9^{\circ}$ Inner and outer contours on maximal nucleus clusters (MNCs) on tessellated digital images [38, §8.9-8.2] form vortex cycles. An open problem is to construct a $\mathrm{CW}$ topology on collections of MNC vortex cycles equipped with the relator $\left\{\begin{array}{c}\text { conn } \begin{array}{c}\text { conn } \\ \text { conn } \\ \delta\end{array}, \delta_{\Phi} \\ \delta\end{array}\right\}$.

open- $10^{\circ}$ An open problem is to construct a Leader uniform topology on a collection of MNC vortex cycles equipped with the relator

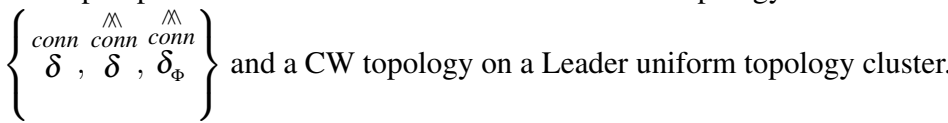

open-11 $1^{o}$ Brain tissue tessellation shows an absence of canonical microcircuits [41]. For related work on donut-like trajectories along preferential brain railways, shaped as a torus, see, e.g., [47]. An open problem is to construct a CW topology on a Leader uniform $\stackrel{M}{M} \stackrel{M}{\text { conn }}$ topology cluster (equipped with the proximity $\stackrel{\operatorname{conn}}{\delta}$ or with $\delta_{\Phi}$ ) that results from a brain tissue tessellation. This is an application of the result from Problem 9.

open-12 $2^{\circ}$ Vortex Cat in spacetime. By tessellating a video frame showing a cat, finding the maximum nucleus cluster MNC on the tessellated frame, and constructing fine and coarse contours surrounding the MNC nucleus, we obtain a vortex cycle. By repeating these steps over a sequence of frames in a video, we obtain a vortex cat cycle in spacetime. See, for example, the sample vortex cat cycles in [9] and [10]. An open problem is the construction of a Leader uniform topology on the collection of video frame vortex cat cycles equipped with the proximity $\stackrel{c o n n}{\delta}$ and to track the persistence of a Leader uniform topology cluster over a video frame sequence.

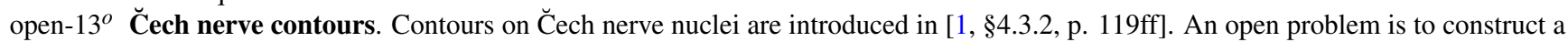
M
conn descriptive CW topology on a collection of C̆ech nerve contours equipped with the proximity $\stackrel{\text { conn }}{\delta_{\Phi}}$.

\section{Acknowledgement}

Many thanks are extended to the anonymous reviewers for their astute, very helpful suggestions and corrections. The research has been supported by the Natural Sciences \& Engineering Research Council of Canada (NSERC) discovery grant 185986, Scientific and Technological Research Council of Turkey (TÜBİTAK) Scientific Human Resources Development (BIDEB) under grant no: 2221-1059B211402463, and Instituto Nazionale di Alta Matematica (INdAM) Francesco Severi, Gruppo Nazionale per le Strutture Algebriche, Geometriche e Loro Applicazioni grant 9920160 000362, n.prot U 2016/000036.

\section{References}

[1] M.Z. Ahmad and J.F. Peters, Proximal C̆ech complexes in approximating digital image object shapes. Theory and application, Theory and Applications of Math. \& Comp. Sci. 7 (2017), no. 2, 81-123, MR3769444.

[2] V.A. Baikov, R.R. Gilmanov, I.A. Taimanov, and A.A. Yakovlev, Topological characteristics of oil and gass reservoirs and their applications, Integrative Machine Learning, LNAI 10344 (A. Halzinger et. al., ed.), Springer, Berlin, 2017, pp. 182-193.

[3] D. Baldomir and P. Hammond, Geometry of electromagnetic systems, Oxford,UK, Clarendon Press, $1996, \mathrm{xi}+239 \mathrm{pp} ., \mathrm{Zbl} 0919.76001$.

[4] J.M.M. Barata, P.J.C.T. Santos N. Bernardo, and A.R.R. Silva, Experimental study of a ground vortex: the effect of the crossflow velocity, 49th AIAA Aerospace Sciences Meeting, AIAA, 2011, pp. 1-9.

[5] J.S. Birman and W.W. Menasco, Studying links via closed braids. V: the unlink, Trans. Amer. Math. Soc. 329 (1992), no. $2,585-606$.

[6] A. Di Concilio, C. Guadagni, J.F. Peters, and S. Ramanna, Descriptive proximities I: Properties and interplay between classical proximities and overlap, arXiv 1609 (2016), no. 06246v1, 1-12, Math. in Comp. Sci. 2017, https ://doi .org/10.1007/s11786-017-0328-y, in press.

[7] , Descriptive proximities. properties and interplay between classical proximities and overlap, Math. Comput. Sci. 12 (2018), no. 1, 91-106, MR3767897.

[8] G.E. Cooke and R.L. Finney, Homology of cell complexes. based on lectures by norman e. steenrod, Princeton University Press; University of Tokyo Press, Tokyo, Princeton, New Jersey, 1967, xv+256 pp., MR0219059.

[9] E. Cui, Video vortex cat cycles part 1, Tech. report, University of Manitoba, Computational Intelligence Laboratory, Deparment of Electrical \& Computer Engineering, U of MB, Winnipeg, MB R3T 5V6, Canada, 2018, https://youtu. be/rVGmkGTm40c.

[10] _ Video vortex cat cycles part 2, Tech. report, University of Manitoba, Computational Intelligence Laboratory, Deparment of Electrical \& Computer Engineering, U of MB, Winnipeg, MB R3T 5V6, Canada, 2018, https: //youtu. be/y JBCdLhgcqk.

[11] A. Dareau, E. Levy, M.B. Aguilera, R. Bouganne, E. Akkermans, F. Gerbier, and J. Beugnon, Revealing the topology of quasicrystals with a diffraction experiment, arXiv, Physical Review Letters 1607 (2017), no. 00901v2, 1-7, doi.org/10.1103/PhysRevLett.119.215304.

[12] I.V. Dzedolik, Vortex properties of a photon flux in a dielectic waveguide, Technical Physics 75 (2005), no. 1, 137-140.

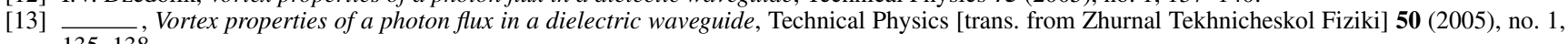
$135-138$.

[14] G. Dvali E. Adelberger and A. Gruzinov, Structured light meets structured matter, Phys. Rev. Letters 98 (2007), 010402-1-010402-4. 
[15] H. Edelsbrunner and J.L. Harer, Computational topology. An introduction, Amer. Math. Soc., Providence, RI, 2010, xii+241 pp. ISBN: 978-0-82184925-5, MR2572029.

[16] M. Fermi, Persistent topology for natural data analysis - a survey, arXiv 1706 (2017), no. 00411v2, 1-18.

[17] Why topology for machine learning and knowledge extraction, Machine Learning \& Knowledge Extraction 1 (2018), no. 6, 1-6, https: //doi.org/10.3390/make1010006.

[18] A. Flammini and A. Stasiak, Natural classification of knots, Proc. R. Soc. Lond. Ser. A Math. Phys. Eng. Sci. 463 (2017), no. 2078, 569-582, MR2288834.

[19] C. Guadagni, Bornological convergences on local proximity spaces and $\omega_{\mu}$-metric spaces, Ph.D. thesis, Università degli Studi di Salerno, Salerno, Italy, 2015, Supervisor: A. Di Concilio, 79pp.

[20] M. Ostavari H. Boomari and A. Zarei, Recognizing visibility graphs of polygons with holes and internal-external visibility graphs of polygons, arXiv 1804 (2018), no. 05105v1, 1-16.

[21] M. Hance, Algebraic structures on nearness approximation spaces, Ph.D. thesis, University of Pennsylvania, Department of Physics and Astronomy, 2015, supervisor: H.H. Williams, vii+113pp.

[22] A. Hatcher, Algebraic topology, Cambridge University Press, Cambridge, UK, 2002, xii+544 pp. ISBN: 0-521-79160-X, MR1867354.

[23] C. Jordan, Cours d'analyse de l'École polytechnique, tome i-iii, Éditions Jacques Gabay, Sceaux, 1991, reprint of 1915 edition, Tome I: MR1188186,Tome II: MR1188187, Tome III: MR1188188.

[24] W. Thomson (Lord Kelvin), On vortex atoms, Proc. Roy. Soc. Edin. 6 (1867), 94-105.

[25] S. Leader, On clusters in proximity spaces, Fundamenta Mathematicae 47 (1959), 205-213.

[26] N.M. Litchinitser, Structured light meets structured matter, Science, New Series 337 (2012), no. 6098, 1054-1055.

[27] _ Structured light meets structured matter, Science, New Series 337 (2012), no. 6098, 1054-1055.

[28] R. Maehara, The Jordan curve theorem via the Brouwer fixed point theorem, Amer. Math. Monthly 91 (1984), no. 10, 641-643, MR0769530.

[29] J.R. Munkres, Topology, $2^{\text {nd }} e d$., Prentice-Hall, Englewood Cliffs, NJ, 2000, xvi + 537 pp., $1^{\text {st }}$ Ed. in 1975,MR0464128.

[30] J.P. Murphy and D.G. MacManus, Ground vortex aerodynamics under crosswind conditions, Experiments in Fluids 50 (2011), no. 1, $109-124$.

[31] M. Pellikka, S. Suuriniemi, and L. Kettunen, Homology in electromagnetic boundary value problems, Boundary Value Problems 2010 (2010), no. 381953, 1-18, doi : 10.1155/2010/381953.

[32] J. F. Peters and E. İnan, Strongly proximal Edelsbrunner-Harer nerves, Proc. Jangjeon Math. Soc. 19 (2016), no. 3, 1-20, MR3618825, zbMATH Zb1 1360.54021 .

[33] J.F. Peters, Local near sets: Pattern discovery in proximity spaces, Math. in Comp. Sci. 7 (2013), no. 1, 87-106, DOI 10.1007/s11786-013-0143-Z, MR3043920, ZBL06156991.

[34] __ Local near sets: pattern discovery in proximity spaces, Math. Comput. Sci. 7 (2013), no. 1, 87-106, MR3043920, ZBL06156991.

[35] Computational proximity. Excursions in the topology of digital images., Intelligent Systems Reference Library 102 (2016), xxviii +433 pp, ISBN: 978-3-319-30260-7; 978-3-319-30262-1; DOI: 10.1007/978-3-319-30262-1; zbMATH Zbl 1382.68008;MR3727129.

[36] _ _ Proximal relator spaces, Filomat 30 (2016), no. 2, 469-472, doi:10.2298/FIL1602469P, MR3497927.

[37] , Two forms of proximal, physical geometry. Axioms, sewing regions together, classes of regions, duality and parallel fibre bundles, Advan. in Math: Sci. J 5 (2016), no. 2, 241-268, Zbl 1384.54015, reviewed by D. Leseberg, Berlin.

[38] Foundations of computer vision. Computational geometry, visual image structures and object shape recognition, Intelligent Systems Ref. Library 124, Springer International Pub. AG, Cham, Switzerland, 2017, xvii+431 pp., ISBN: 978-3-319-52483-2; 978-3-319-52481-8; DOI 10.1007/9783-319-52483-2; MR3769444.

[39] _ Proximal planar shape signatures. Homology nerves and descriptive proximity, Advan. in Math: Sci. J 6 (2017), no. 2, 71-85, Zbl 06855051.

[40] , Proximal planar shapes. Correspondence between shape and nerve complexes, arXiv 1708 (2017), no. 04147v1, 1-12, Bulletin of the Allahabad Math. Soc., Dharma Prokash Gupta Memorial Volume, 2018 in press.

[41] J.F. Peters, A. Tozzi, and S. Ramanna, Brain tissue tessellation shows absence of canonical microcircuits, Neuroscience Letters 626 (2016), 99-105, http://dx.doi.org/10.1016/j.neulet.2016.03.052.

[42] A.K. Travin P.R. Spalart, M. Kh. Strelets and M.L. Slur, Modeling the interaction of a vortex pair with the ground, Fluid Dynamics 36 (1999), no. 6, 899-908

[43] A.R.R. Silva, D.F.G. Dur ao, J.M.M. Barata, P. Santos, and S. Ribeiro, Laser-doppler analysis of the separation zone of a ground vortex flow, 14th Symp on Applications of Laser Techniques to Fluid Mechanics, Lisbon, Portugal, Universidade Beira Interior, 2008, pp. 7-10.

[44] Ju. M. Smirnov, On proximity spaces, American Math. Soc. Translations, Series 2, vol. 38 (AMS, ed.), Amer. Math. Soc., Providene, RI, 1964, pp. 3-36.

[45] Á Száz, Basic tools and mild continuities in relator spaces, Acta Math. Hungar. 50 (1987), no. 3-4, 177-201, MR0918156.

[46] S. De Toffoli and V. Giardino, Forms and roles of diagrams in knot theory, Erkenntnis 79 (2014), no. 4, 829-842, MR3260948.

[47] A. Tozzi, J.F. Peters, and E. Deli, Towards plasma-like collisionless trajectories in the brain, Neuroscience Letters 662 (2018), $105-109$.

[48] E. Čech, Topological spaces, John Wiley \& Sons Ltd., London, 1966, fr seminar, Brno, 1936-1939; rev. ed. Z. Frolik, M. Katĕtov. Scientific editor, Vlastimil Pt 'ak. Editor of the English translation, Charles O. Junge Publishing House of the Czechoslovak Academy of Sciences, Prague; Interscience Publishers John Wiley \& Sons, London-New York-Sydney 1966893 pp., MR0211373.

[49] H. van Leunen, The hilbert book model project, Tech. report, Deparment of Applied Physics, Technische Universiteit Eindhoven, 2018, https: //www.researchgate.net/project/The-Hilbert-Book-Model-Project.

[50] O. Veblen, Theory on plane curves in non-metrical analysis situs, Transactions of the American Mathematical Society 6 (1905), no. 1, 83-98, MR1500697.

[51] J.H.C. Whitehead, Combinatorial homotopy. I., Bulletin of the American Mathematical Society 55 (1949), no. 3, 213-245, Part 1, MR0030759.

[52] S. Willard, General topology, Dover Pub., Inc., Mineola, NY, 1970, xii + 369pp, ISBN: 0-486-43479-6 54-02, MR0264581.

[53] G.M. Ziegler, Lectures on polytopes, Springer, Berlin, 2007, $\mathrm{x}+370$ pp. ISBN: 0-387-94365-X, MR1311028. 